\title{
Adsorption and Nonadiabatic Processes in the Photodesorption of Molecular Oxygen from the Reduced $\mathrm{TiO}_{2}(110)$ Surface
}

\author{
María Pilar de Lara-Castells, ${ }^{\mathrm{a}, *}$ Alexander O. Mitrushenkov, ${ }^{\mathrm{b}}$ Octavio Roncero, ${ }^{\mathrm{a}}$ and Jeffrey L. Krause ${ }^{\mathrm{c}}$ \\ anstituto de Matemáticas y Física Fundamental (C.S.I.C.), Serrano 123, E-28006-Madrid, Spain \\ ${ }^{b}$ Dipartimento di Chimica Fisica ed Inorganica, University of Bologna, Viale Rigorgimento 4, 40136 Bologna, Italy \\ 'Quantum Theory Project, Department of Chemistry and Physics, University of Florida, Gainesville, Florida 32611-8435, USA
}

(Received 14 May 2004 and in revised form 28 June 2004)

\begin{abstract}
We review the adsorption and desorption of molecular oxygen on a reduced $\mathrm{TiO}_{2}(110)$ surface. This system is known to play a fundamental role in heterogeneous photocatalysis. Periodic calculations are performed with the objective of characterizing the variety of stable species of $\mathrm{O}_{2}$ that are known to exist on the $\mathrm{TiO}_{2}$ surface. The implications of our results for recent experiments are discussed. We also consider a direct optical excitation mechanism for the ultraviolet (UV) light-desorption process and model the most stable $\mathrm{O}_{2} / \mathrm{TiO}_{2-x}$ system as a cluster. High-level ab initio calculations of the excited states and interaction matrix elements are performed using different orbitals, separately optimized for the target states. The nonadiabatic and dipole-moment couplings are calculated directly from the correlated wave functions by a special transformation to bi-orthonormal (dual) orbital sets to preserve their structure. The method used for the electronic structure calculations is described in detail. Finally, the effect of the electronic coupling in the UVphotodesorption dynamics is analyzed in detail.
\end{abstract}

\section{INTRODUCTION}

A wealth of experimental data now exists on quantumstate-selective, light-induced desorption of molecules from metal and metal oxide surfaces. Most previous calculations on these systems use empirical potential energy surfaces, which cannot capture all of the relevant physics. One important step in the development of more realistic models is the study of molecule-surface interactions using ab initio calculations to characterize not only the important regions of the potential but also the molecule-surface nonadiabatic and electronic couplings. However, excited states and couplings for systems consisting of adsorbates on metals and metal oxides are difficult to compute in full generality.

In this work, we address the problem by focusing on a system consisting of molecular oxygen adsorbed on a titanium dioxide surface, specifically the reduced $\mathrm{TiO}_{2}$ (110) rutile surface. Titanium dioxide is employed in a diverse range of applications (for a recent review see, for example, ref 1). As a result, $\mathrm{TiO}_{2}$ is by far the best- characterized metal oxide surface. In powder form, it is used in industry as a white pigment and opacifier in paints and cosmetics. $\mathrm{TiO}_{2}$ is also used widely as a catalyst support, in gas sensors, in solar cells, and as a biocompatible interface for medical implants. In the area of photocatalytic degradation, ${ }^{2-6} \mathrm{TiO}_{2}$ has found applications in air purification, self-cleaning surfaces, and transparent superhydrophilic coatings. This is mainly due to the strong oxidizing power of $\mathrm{TiO}_{2}$ under UV illumination, and its chemical stability. ${ }^{5}$

The photocatalytic process is believed to begin with the creation of electron-hole pairs by UV illumination of the $\mathrm{TiO}_{2}$ surface. The photoelectrons may be captured by molecular oxygen, thus avoiding electron-hole recombination and the accumulation of negative charge on the surface. ${ }^{2,7,8}$ In addition, the photogenerated holes may be captured by water molecules to produce hydroxyl radicals that, presumably, can initiate the oxida-

*Author to whom correspondence should be addressed. E-mail: delara@imaff.cfmac.csic.es

$$
\text { Israel Journal of Chemistry Vol.45 } 2005 \quad \text { pp.59-76 }
$$


tion of organic molecules. However, some studies have suggested that activated molecular oxygen itself may initiate photooxidation reactions. ${ }^{4-6}$ The scavenging process of molecular oxygen has been shown to resemble the reaction of $\mathrm{O}_{2}$ with thermally generated electronic defects on $\mathrm{TiO}_{2}{ }^{7}$ For this reason, the system consisting of $\mathrm{O}_{2}$ adsorbed on a reduced $\mathrm{TiO}_{2}$ (110) surface (on which oxygen vacancy sites are the defects) has been studied extensively by experimentalists using techniques such as temperature-programmed desorption ${ }^{7,9}$ (TPD), controlled photodesorption (PD), ${ }^{4,10}$ and, very recently, time-resolved scanning tunneling microscopy (STM). ${ }^{11,12}$

Experiments by Yates and coworkers ${ }^{4,10}$ detect the presence of adsorbed oxygen by controlled photodesorption (PD) after $\mathrm{O}_{2}$ is allowed to interact with the reduced $\mathrm{TiO}_{2}(110)$ surface at $100 \mathrm{~K}$. In measurements of photodesorption of $\mathrm{O}_{2}$ and photooxidation of $\mathrm{CO}$, the experiments observe an unusual dependence of the desorption and oxidation rate on temperature. Based on a characteristic kinetic profile, they distinguish two species of adsorbed $\mathrm{O}_{2}$. The species at lower temperature (referred to as the $\alpha$ species) can photooxidize $\mathrm{CO}$ to $\mathrm{CO}_{2}$. This species experiences a slower photodesorption and, in turn, can be resolved into two distinct species. ${ }^{10}$ Heating to $250 \mathrm{~K}$ transforms these species irreversibly to the species at high temperature (referred to as the $\beta$ species). The $\beta$ species experiences faster photodesorption and is not able to photooxidize $\mathrm{CO}$ to $\mathrm{CO}_{2}$. When the surface is heated to $400 \mathrm{~K}$, no molecular oxygen is detected, due, presumably, to a dissociative filling of the vacancies. Additional experiments on UVinduced desorption by Möller et al., ${ }^{13}$ measure time-offlight (TOF) spectra of the desorbed oxygen molecules and the dependence of the desorption signal on the laser intensity. The TOF spectra display two components (a fast one and a slow one) and the authors suggest that the fast signal is due to photoelectronic activation of $\mathrm{O}_{2}^{-}$ radicals adsorbed on defect sites.

In complementary experiments, Henderson et al., ${ }^{7,9}$ using different experimental techniques such as TPD, electron energy loss spectroscopy (EELS), and highenergy (vibrational) electron energy (HREELS), examine the interaction of $\mathrm{O}_{2}$ with the reduced $\mathrm{TiO}$ (110) surface at low temperature $(120 \mathrm{~K})$. They show that $\mathrm{O}_{2}$ is adsorbed in molecular form on the vacancies if the adsorption temperature is below $150 \mathrm{~K}$, otherwise it adsorbs dissociatively. The spectroscopic evidence indicates that, after adsorption at $120 \mathrm{~K}$, adsorbed molecules are present on the surface as $\mathrm{O}_{2}^{-}$species located at oxygen vacancy sites. These species are stable up to $410 \mathrm{~K}$, at which point they desorb. ${ }^{7}$ Henderson et al. also observe a weak TPD peak at $145 \mathrm{~K}$ due to a physisorption state of molecular oxygen when the saturation coverage is about three times the vacancy concentration (8\%). Further studies by Perkins and Henderson ${ }^{9}$ suggest that the $410 \mathrm{~K}$ TPD peak stems from the $\alpha$ species detected by Yates. More recently, Schaub et al. ${ }^{12}$ and Wahlström et al., ${ }^{11}$ by means of time-resolved STM, detect $\mathrm{O}_{2}$ molecules residing on fivefold (5f) coordinated $\mathrm{Ti}$ atoms adjacent to the vacancies. The experimental data are interpreted with a model in which oxygen diffuses along the [001] direction at temperatures ranging from 170 to $250 \mathrm{~K}$.

From a theoretical perspective, the main difficulty in describing the $\mathrm{O}_{2} / \mathrm{TiO}_{2-x}$ system is due to the fact that the $\mathrm{O}_{2}$ ground state is an open shell system (a triplet state). In response to the unpaired electrons in $\mathrm{O}_{2}$, the electron density in the vicinity of the vacancy can adopt a spinpaired as well as a spin-unpaired configuration. Depending on the electron spin-state in the surface, an oxygen molecule can adsorb in either a singlet, triplet, or quintet state. Further complicating the theoretical description is the observation that molecular oxygen adsorbs at both the vacancy and the $\operatorname{Ti}(5 \mathrm{f})$ sites. This makes the design of an appropriate cluster model difficult. ${ }^{14}$ For this reason, and to obtain relative adsorption energies for the various spin and spatial configurations of the adsorbate-surface system reliably, we chose to include the periodicity explicitly. Recently, we reported the first theoretical analysis of the $\mathrm{O}_{2} / \mathrm{TiO}_{2-x}(110)$ interaction based on periodic ab initio calculations. ${ }^{15,16}$ The results of these studies will be reviewed in this work.

A variety of mechanisms have been proposed to explain the photodesorption data. One possibility is an indirect process involving the photodetachment of a substrate electron which interacts with the adsorbate to cause desorption. ${ }^{17,18}$ Another is excitation of electronhole pairs and subsequent energy transfer to lattice vibrations, thus producing thermal desorption. ${ }^{19}$ These mechanisms may be dominant in metals but are probably not important for metal oxide and semiconductor surfaces. More likely in these cases are either the excitation of adsorbate modes,${ }^{20}$ followed by energy transfer and desorption, or the direct excitation and breaking of adsorbate-substrate bonds. ${ }^{21,22}$

To model the photodesorption process reliably, accurate potential energy surfaces and couplings are required. However, current ab initio methods are unable to treat extended adsorbate-surface systems reliably. One way to proceed is to use one-dimensional lattices as models of the surface. This allows the use of accurate methods for the calculation of excited electronic states. ${ }^{23}$ For systems with more than one periodic dimension, though, current methods are limited to either Hartree- 
Fock (HF) or Density Functional Theory (DFT). While such methods are reliable for ground states, they provide, in general, poor descriptions of excited states.

The alternative to full quantum treatments of the surface is to use highly accurate ab initio methods within a cluster model, in which the substrate is "truncated" to a number of atoms, or cluster, whose size is limited by the accuracy required and the computational cost. The most obvious problem of this method is the breaking of bonds at the edges of the cluster, which would generate uncoupled orbitals, known as "dangling bonds". ${ }^{24}$ To avoid this problem, the dangling bonds are saturated with hydrogen atoms, or the cluster is embedded in a semi-infinite array of point charges to simulate the Madelung field at the edges of the cluster. More sophisticated techniques have been applied to adsorbates on metal surfaces using an embedding potential to describe the interaction between the cluster and the extended condensed phase (see, for example, ref 25).

For the photodesorption of adsorbates from insulator surfaces, recent work by Klüner et al. ${ }^{26}$ has shown that the use of point-charge cluster models with extensions to configuration interaction (CI) methods to calculate excited states can provide results in excellent agreement with experiment. In this work, we use a hydrogen-saturated cluster model and high-level ab initio quantum chemical methods to compute both the excited states and couplings. The method is rendered computationally feasible by using different orbitals, separately optimized for each interacting electronic state. We describe a computational algorithm to calculate the dipole, electronic, and nonadiabatic couplings using a transformation to biorthonormal basis. The approach is applied to a calculation of the low-lying excited states, dipole moments, and nonadiabatic couplings of the $\mathrm{O}_{2} / \mathrm{TiO}_{2-x}$ system. To do this, a cluster is designed to obtain good agreement between periodic and cluster model results for the most stable species calculated by the extended procedure (and observed experimentally).

In the following section, we review the periodic calculations for the $\mathrm{O} / \mathrm{TiO}_{2-x}$ system. Next, the theoretical details of the cluster calculations for excited-state energies and nonadiabatic coupling elements are presented. In Section 3, we focus on results for the UV-photoinduced desorption process including nonadiabatic effects. Finally, we conclude with a summary of the main results, and plans for future work.

\section{ADSORPTION OF $\mathrm{O}_{2}$ ON THE REDUCED TIO (110) SURFACE: A PERIODIC MODEL}

The periodic calculations were performed with the CRYSTAL98 code $^{27}$ at the unrestricted Hartree-Fock level of theory. This method is well-established for studies of bulk, ${ }^{28}$ clean, ${ }^{29}$ and reduced surfaces ${ }^{30}$ of $\mathrm{TiO}_{2}$, and the adsorption of various molecules on the $\mathrm{TiO}_{2}$ surface (see, e.g., ref 31).

To simulate the (110) surface, we use a 2-dimensional periodic slab model with a thickness of nine layers. The slab geometry is modeled as periodic in the [001] and [110] directions, but finite in the [110] direction. We study only the reduced surface that is created by removal of alternate bridging oxygen atoms in the [001] direction, which have the weakest binding energy to the surface. Neutral oxygen atoms only are removed from the model surfaces, (not the $\mathrm{O}^{2-}$ ions), so the unit cell remains neutral in the periodic calculation.

For the description of the reduced surface, convergence with respect to the size of the unit cell is checked carefully. The surface unit cell is doubled in the crystallographic [001] direction, and either single or tripled in the [110] direction, creating $2 \times 1$ and $2 \times 3$ supercells (see Fig. 1), respectively. The former models a reduced surface with a defect surface of one-half of a monolayer while the latter has $16 \%$ vacancies. In the experiments by Henderson et al., 7 the defect density was $8 \%$, so the larger supercell was chosen to allow direct comparison with experiment. The model surface is then covered with $\mathrm{O}_{2}$ molecules in a ratio of $1: 1$ or $3: 1$ with respect to the vacancies. Positions of the surface ions, oxygen ions belonging to the third and fourth layers, the bond lengths of $\mathrm{O}_{2}$, and the $\mathrm{O}_{2}$-substrate distances are optimized using a numerical conjugate gradient method $^{32}$ to a tolerance of $0.01 \AA$ in atomic positions and $10^{-5} \mathrm{eV}$ in total energy. During optimization, the symmetry is reduced to retain only the plane perpendicular to the surface in the [001] direction.

A basis set of contracted Gaussian type orbitals (GTOs) is used to span the Bloch orbitals of the crystal. Due to the large number of atoms per supercell and the low symmetry in the model, the number of required integral evaluations is extremely large. To reduce the cost without loss of accuracy, we use effective core potentials (ECP). For the oxygen atom, we use a Durand-Bartheland ${ }^{33} \mathrm{ECP}$, which replaces the $1 \mathrm{~s}^{2}$ electrons, and a $[6 s 6 p / 2 s 2 p]$ basis set for the valence electrons. For the titanium atoms, we use an ECP that includes the $1 \mathrm{~s}^{2}$ to $2 \mathrm{p}^{6}$ electrons in the core, ${ }^{34}$ and a [5s5p3d/2s 2p1d] basis set for the valence electrons. These basis sets have been optimized in previous studies of bulk $\mathrm{TiO}_{2}$ and surfaces of $\mathrm{TiO}_{2},{ }^{28,30,35}$ which are presented elsewhere. ${ }^{28,35}$ The thresholds for integral calculations are set to the values (5 555 10). The convergence thresholds for the eigenvalues and total energies are set to $10^{-9}$ and $10^{-6}$, respectively. The reciprocal space integration uses the Monkhorst-Pack sampling. ${ }^{36}$ 


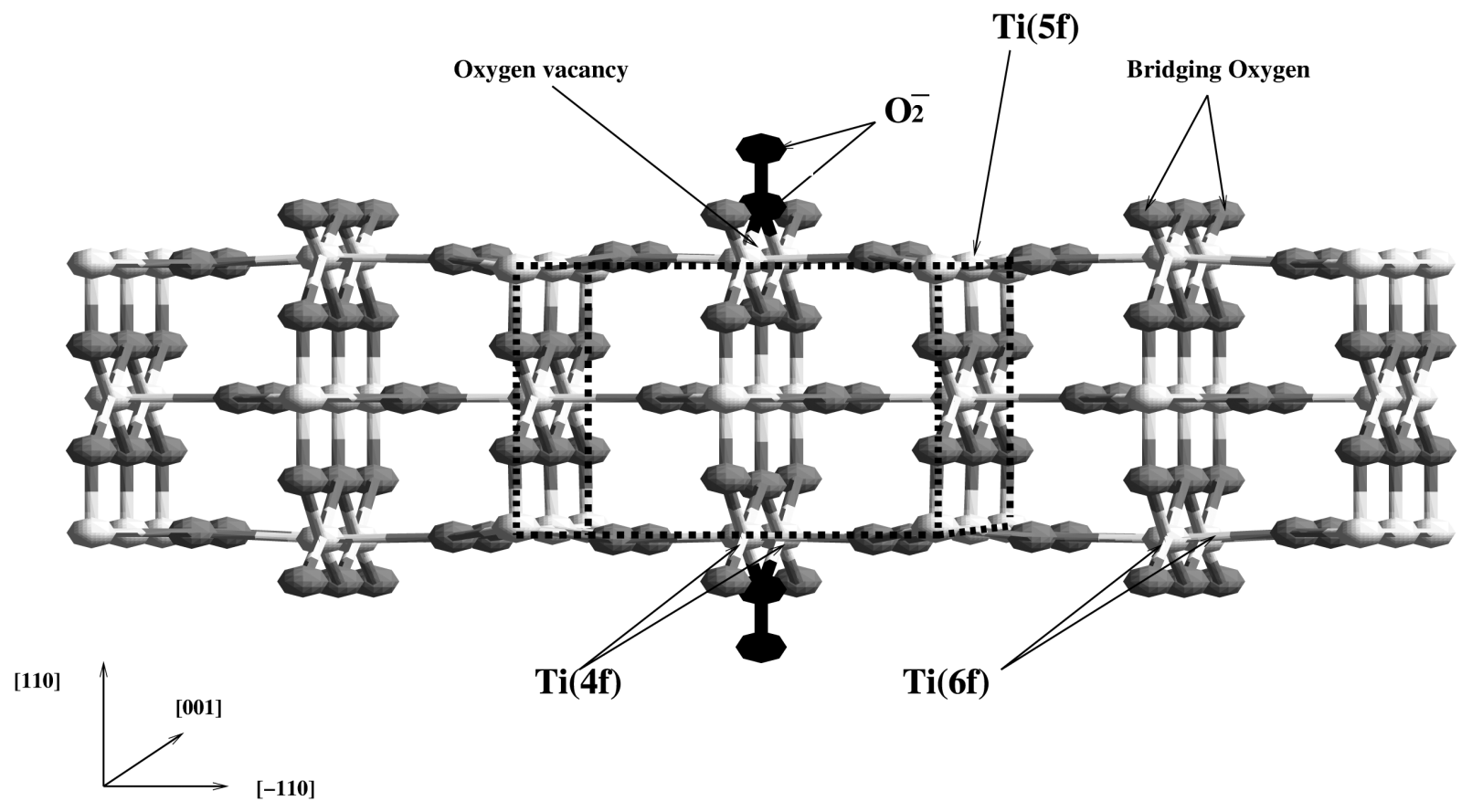

Fig. 1. Side view of the relaxed geometries calculated for the most stable $\mathrm{O}_{2} / \mathrm{TiO}_{2-x}$ system when only one $\mathrm{O}_{2}$ molecule per vacancy is adsorbed. Gray and white spheres indicate oxygen and titanium ions, respectively, and black spheres indicate the oxygen atoms of the adsorbed $\mathrm{O}_{2}$ molecule. Dashed lines represent the $2 \times 1$ supercell slab that models a reduced $\mathrm{TiO}_{2}$ surface with $50 \%$ vacancies, while the $2 \times 3$ supercell slab models surface with $16 \%$ vacancies. The results are from ref 16 ; reprinted with permission from Elsevier.

The wave functions are determined using nine k-points in the irreducible part of the Brillouin zone.

The adsorption energies are calculated with the expression,

$$
E_{\text {ads }}=\left(n E\left[\mathrm{O}_{2}\right]+E\left[\mathrm{TiO}_{2}\right]-E\left[n \mathrm{O}_{2} / \mathrm{TiO}_{2}\right]\right) / n
$$

where $E\left[\mathrm{O}_{2}\right]$ is the energy of the isolated $\mathrm{O}_{2}$ molecule in its equilibrium position, $E\left[\mathrm{TiO}_{2}\right]$ is the energy of the slab that models the reduced surface, $E\left[n \mathrm{O}_{2} / \mathrm{TiO}_{2}\right]$ is the total energy of the slab containing both the surface and the adsorbates, and $n$ is the total number of adsorbed $\mathrm{O}_{2}$ molecules. We checked the convergence of the adsorption energies with respect to the thickness of the slab. The tests showed that $E_{\text {ads }}$ is converged to within $10 \%$ of the value obtained with a 15 -layer slab.

The theoretical method has been tested by calculations of an isolated oxygen molecule, the bulk, and the reduced $\mathrm{TiO}_{2}$ (110) surface. The relative error in the bond length of isolated $\mathrm{O}$ is $0.5 \%$, compared to experiment. The optimized lattice parameters for tetragonal bulk rutile are $a=b=4.509 \AA, c=3.017 \AA$, and $\mathrm{u}=$ 0.305 , which differ from the experimental values ${ }^{37}$ by $-1.69 \%, 2.13 \%$, and $0.06 \%$, respectively.

\subsection{The Reduced $\mathrm{TiO}_{2}$ (110) Surface}

The most relevant conclusions from the results on the reduced (110) surface are: (a) The excess electrons are trapped in localized states that consist, basically, of $3 \mathrm{~d}$ orbitals of Ti cations, with energies between the conduction and the valence bands; (b) The spin density due to the excess electrons is spread over both the superficial cations of Ti and those in internal layers. Also, an analysis of the distribution of charge density reveals that most of the charge from the neutral $\mathrm{O}$ atom that leaves the surface is gained by one of the superficial Ti cations nearest to the vacancy and one of the metallic ions in the central layer. These conclusions agree with previous results from Lindan et al., ${ }^{38}$ who used a very different theoretical treatment consisting of plane-wave basis sets and a gradient-corrected DFT approximation. As can be seen in Table 1, our results for the ionic displacements due to relaxation of the reduced surface are in reasonable agreement with the results calculated by Lindan et al. The largest difference is found in the value corresponding to the displacement of the bridging oxygen along the [001] direction (see Fig. 2). However, this displacement is associated with a very soft mode, as 
Table 1. Ionic displacements due to relaxation of the reduced (110) $2 \times 1$ surface, from ref 15 . Labels refer to Fig. 2 . The displacements are in $\AA$ and are derived from the bulk terminated positions (data in parenthesis are from ref 38)

\begin{tabular}{lccc}
\hline label & {$[001]$} & {$[\overline{1} 10]$} & {$[110]$} \\
\hline 1 & 0.15 & & -0.02 \\
& $(0.04)$ & & $(-0.02)$ \\
2 & -0.02 & -0.16 \\
& & & $(-0.13)$ \\
3 & -0.02 & -0.03 \\
& & & $(-0.09)$ \\
4 & 0.03 & & 0.02 \\
& $(0.05)$ & & $(0.03)$ \\
5 & -0.16 & & -0.03 \\
& $(-0.17)$ & & $(-0.05)$ \\
6 & 0.02 & -0.03 & 0.25 \\
& & $(-0.05)$ & $(0.25)$ \\
7 & 0.02 & 0.03 & 0.25 \\
& & $(0.05)$ & $(0.25)$ \\
8 & 0.05 & -0.04 & 0.25 \\
& $(0.03)$ & & $(0.25)$ \\
9 & 0.05 & 0.04 & 0.25 \\
& $(0.03)$ & & $(0.25)$ \\
\hline
\end{tabular}

noted previously by Harrison et al. ${ }^{38}$ in a study of the (110) $\mathrm{TiO}_{2}$ surface. For this reason, convergence of this displacement is difficult. Test calculations showed that most of the ionic displacements are converged to within $0.02 \AA$ of the values obtained with a slab fifteen layers thick but, once again, there was a large difference in the value corresponding to the bridging oxygen. ${ }^{15}$

Table 1 and Fig. 2 show that large ionic displacements occur in the regions near the vacancy. In particular, the two adjacent under-coordinated $\mathrm{Ti}(4 \mathrm{f})$ cations move in the [001] direction by 0.03 and $-0.16 \AA$, respectively, the two adjacent bridging $\mathrm{O}$ atoms move down, the oxygen below the vacancy moves out by $0.15 \AA$, and the coplanar oxygen ions experience a very large outward relaxation of $0.25 \AA$. The main cause of these displacements is strengthened bonds between the under-coordinated Ti(4f) ions and the adsorbed oxygen. The charge redistribution causes a distortion of the symmetry that makes the $\mathrm{Ti}(4 \mathrm{f})$ ions inequivalent. As a result, the cation that gains more electron density moves less toward the bridging oxygen atom than its counterpart $(0.03$ vs. $0.16 \AA)$. This is due to a reduction of coordination because the atomic oxygen vacancy is

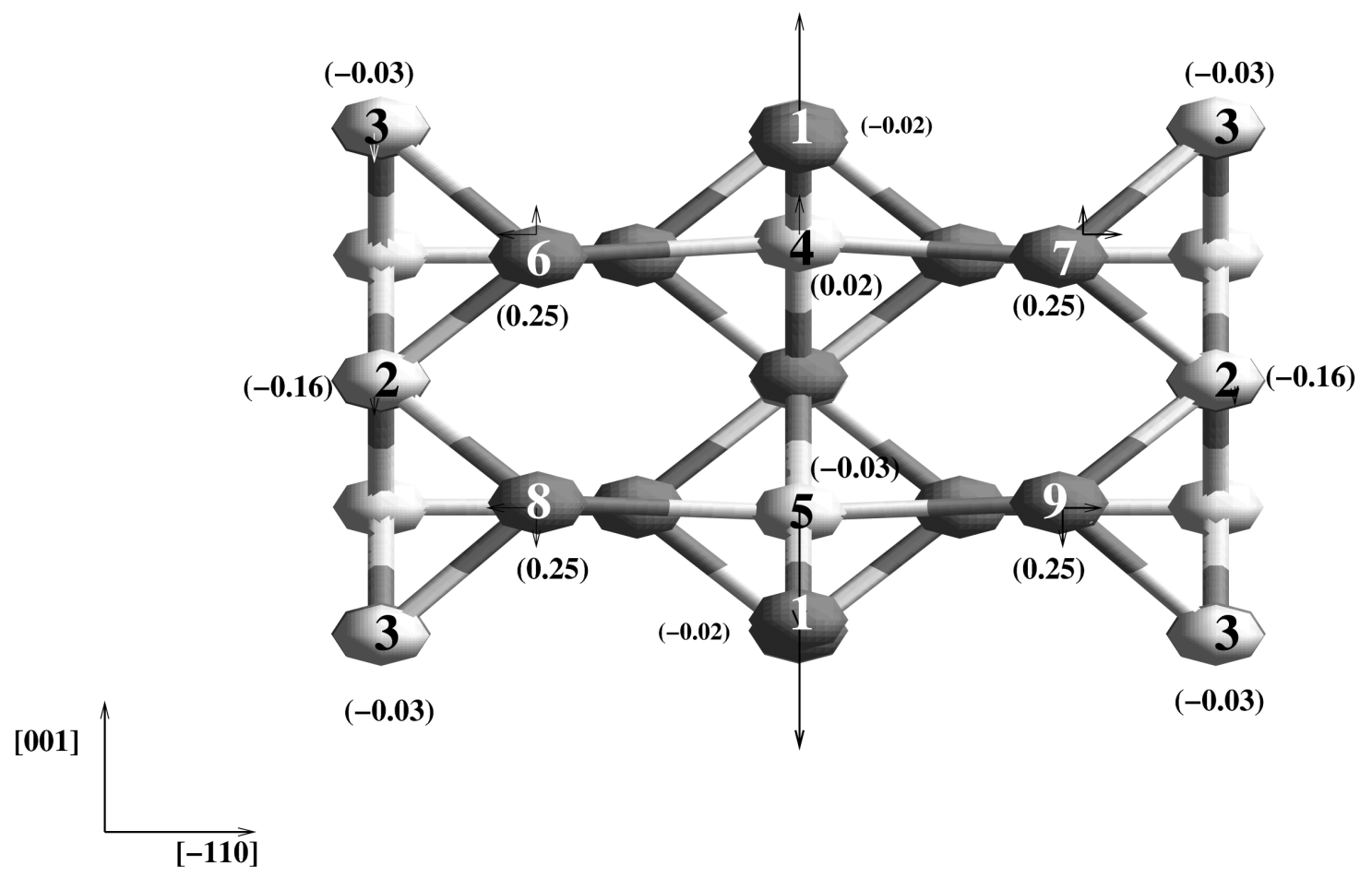

Fig. 2. View of the $2 \times 1$ surface cell along the surface normal direction. Gray and white spheres indicate oxygen and titanium ions, respectively. The arrows indicate the directions of the ionic displacements and are approximately to scale. The displacements along the surface normal are indicated in parenthesis. The results are from ref 16; reprinted with permission from Elsevier. 
compensated by the reduction of the total charge of the ion.

Also, in agreement with previous calculations, the ground state of the reduced surface with 50\% vacancies (the $2 \times 1$ supercell) is a triplet spin-symmetry state. In contrast, for the reduced surface with $16 \%$ vacancies (the $2 \times 3$ supercell), we find that the antiferromagnetic arrangement of the vacancy electrons is lower in energy than the ferromagnetic arrangement. However, the energy difference between the triplet and singlet spinsymmetry states is also smaller $(0.22$ vs. $-2.40 \mathrm{eV})$. These results indicate that the reduced surface contains a large number of local energy minima nearly degenerate.

\subsection{Adsorption of $\mathrm{O}_{2}$ on the Reduced $\mathrm{TiO}_{2}(110)$ Surface}

\subsubsection{Chemisorption at the Vacancy}

For the case of chemisorption, the most stable species is a triplet state in which $\mathrm{O}$ absorbs on the vacancy in a perpendicular arrangement as a superoxide radical anion $\mathrm{O}_{2}^{-}$(see Fig. 1). This is consistent with Mulliken population analysis, which shows that the net charge on the adsorbed species is nearly -1 (see Table 2). The equilibrium bond length $(1.369 \AA)$ is also nearly the same as the bond length of an isolated $\mathrm{O}_{2}^{-}$anion (1.349 $\AA$ ). When this species forms, only one of the reduced electrons is transferred to the adsorbed $\mathrm{O}_{2}$, while the other electron remains in the subsurface region. As a result, the reoxidation of the vacancy is not complete and the surface remains active for the adsorption of additional $\mathrm{O}_{2}$ molecules. The value of the adsorption energy $(3.39 \mathrm{eV})$ indicates that this species is strongly bound, corresponding to a chemisorption state. When the oxygen rotates parallel to the surface, the $\mathrm{O}_{2}$ bond breaks. This suggests that the perpendicular mode is quite rigid.

As mentioned previously, we checked the convergence of the results with respect to the slab size by performing calculations of the reduced $\mathrm{TiO}_{2}(110)$ surface using $2 \times 1$ and $2 \times 3$ supercells. The results of this comparison are shown in Table 2. In general, the values for the two cases are rather similar. This is a strong indication that the adsorption mechanism is localized near the defect. The atomic positions are closer to the corresponding ones in the defect-free case than to those in the reduced surface due to the fact that the adsorption of one $\mathrm{O}_{2}$ molecule gives rise to a partial restoration of the under-coordination of the Ti(4f) ions. For example, in the $(2 \times 3)$ supercell, the displacements of the two adjacent $\mathrm{Ti}(4 \mathrm{f})$ cations in the [001] direction $(0.058 \AA)$ are lower than those in the reduced surface $(0.10$ vs. $-0.14 \AA$ ), and the coplanar oxygen atoms experience an
Table 2. Summary of the results from calculations of $\mathrm{O}_{2}$ adsorbed at the vacancy in a triplet state in perpendicular (T) and parallel (P) orientations to the surface (from refs 15 and 16). In the $\mathrm{T}$ configuration, the superscript $a$ refers to the atom of the $\mathrm{O}_{2}$ molecule oriented towards the surface, the superscript $b$ to the atom further from the surface, and the superscript $c$ to the reduced Ti cation localized in the central layer. Data in parentheses are from model calculations on a $(2 \times 3)$ supercell

\begin{tabular}{lcc}
\hline parameter & $\mathrm{T}$ & $\mathrm{P}$ \\
\hline Binding energy $(\mathrm{eV})$ & 3.39 & 1.44 \\
& $(3.61)$ & \\
O-O distance in adsorbed $\mathrm{O}_{2}(\AA)$ & 1.369 & 2.071 \\
& $(1.371)$ & \\
Length of isolated $\mathrm{O}_{2}$ bond $(\AA)$ & 1.197 & 1.197 \\
Length of isolated $\mathrm{O}_{2}^{-1}$ bond $(\AA)$ & 1.349 & 1.349 \\
Spin density for $\mathrm{O}_{\text {prox }}{ }^{a}$ & -0.014 & 1.016 \\
& $(-0.022)$ & \\
Spin density for $\mathrm{O}_{\text {dist }}{ }^{b}$ & 1.018 & 1.016 \\
& $(1.026)$ & \\
Spin density for $\mathrm{Ti}^{c}$ & 0.990 & $\ldots$ \\
& $(0.985)$ & \\
Mulliken charge for $\mathrm{O}_{\text {prox }}{ }^{a}$ & -0.938 & -0.831 \\
& $(-0.959)$ & \\
Mulliken charge for $\mathrm{O}_{\text {dist }}{ }^{b}$ & 0.048 & -0.831 \\
& $(0.071)$ & \\
Electron transfer for Tic & -0.456 & $\ldots$ \\
& $(-0.413)$ & \\
Distance of $\mathrm{O}_{\text {prox }}{ }^{a}$ to the surface $(\AA)$ & 1.269 & 1.136 \\
& $(1.272)$ & \\
Distance of $\mathrm{O}_{\text {bridging }}$ to the surface $(\AA)$ & 1.077 & 0.968 \\
& $(1.076)$ & \\
\hline
\end{tabular}

outward shift that is much less pronounced (0.092 vs. $0.25 \AA$ ) and is closer to the displacement in the stoichiometric surface $(0.11 \AA)$. Finally, the oxygen atom below the vacancy moves out of the surface by almost half the shift on the reduced surface ( 0.064 vs. $0.15 \AA$ ).

The next most stable species is assigned as $\mathrm{O}_{2}^{2-}$ and corresponds to a singlet spin-symmetry state (see Table 3). Note that the charge on the peroxo $\mathrm{O}_{2}^{2-}$ species adsorbed in a perpendicular approach, -1.561 , is polarized towards the $\mathrm{O}$ atom closer to the surface $(-1.289$ vs. $-0.274)$, which, in turn, has a strong overlap with the $\mathrm{Ti}(4 \mathrm{f})$ center $(0.077)$ very near to the bridging $\mathrm{O}$ atom and the same Ti site (0.095). These data indicate that this species is a clear precursor to dissociative filling of the vacancies. In contrast to the triplet state, in which the $\mathrm{O}_{2}^{-}$species rotates parallel to the surface, and perpendicular to the line connecting the two $\operatorname{Ti}(4 \mathrm{f})$ cations, the binding energy is the same within numerical accuracy $(1.96 \mathrm{eV})$. Again in contrast to the triplet case, the value of the adsorption energy obtained from calculations 
Table 3. Summary of the results from calculations of $\mathrm{O}_{2}$ adsorbed at the vacancy in a singlet state and different spatial configurations: perpendicular to the surface $(\mathrm{T})$; parallel to both the surface and the $\operatorname{Ti}(4 \mathrm{f})-\operatorname{Ti}(4 \mathrm{f})$ axis $\left(\mathrm{P}_{2}\right)$; paralel to the surface and perpendicular to the $\operatorname{Ti}(4 \mathrm{f})-\mathrm{Ti}(4 \mathrm{f})$ axis $\left(\mathrm{P}_{1}\right)$, from refs 15 and 16. In the $\mathrm{T}$ configuration, the superscript $a$ refers to the atom of the $\mathrm{O}_{2}$ molecule oriented towards the surface, and the superscript $b$ to the atom further from the surface. Data in parentheses are from model calculations on a $(2 \times 3)$ supercell, from ref 16

\begin{tabular}{lccc}
\hline parameter & $\mathrm{T}$ & $\mathrm{P}$ & $\mathrm{P}$ \\
\hline Binding energy $(\mathrm{eV})$ & 1.96 & 1.96 & 0.99 \\
& $(3.12)$ & & \\
O-O distance in adsorbed $\mathrm{O}_{2}(\AA)$ & 1.609 & 1.465 & 1.660 \\
& $(1.615)$ & & \\
Length of isolated $\mathrm{O}_{2}$ bond $(\AA)$ & 1.197 & 1.197 & 1.197 \\
Length of isolated $\mathrm{O}_{2}^{-1}$ bond $(\AA)$ & 1.349 & 1.349 & 1.349 \\
Mulliken charge for $\mathrm{O}_{\text {prox }}{ }^{a}$ & -1.289 & -0.803 & -0.729 \\
& $(-1.293)$ & & \\
Mulliken charge for $\mathrm{O}_{\text {dist }}{ }^{b}$ & -0.274 & -0.803 & -0.729 \\
& $(-0.280)$ & & \\
Distance of $\mathrm{O}_{\text {prox }}{ }^{a}$ to the surface $(\AA)$ & 1.020 & 1.187 & 1.627 \\
& $(1.040)$ & & \\
Distance of $\mathrm{O}_{\text {bridging }}$ to the surface $(\AA)$ & 1.000 & 0.983 & 0.934 \\
& $(1.019)$ & & \\
\hline
\end{tabular}

with the $(2 \times 3)$ slab is rather different than that calculated with the $(2 \times 1)$ slab $(3.12$ vs. $1.96 \mathrm{eV})$. This is probably due to the fact that the formation of this species requires the diffusion of excess electron density from the subsurface region to the surface, which is not a localized process.

\subsubsection{Adsorption on the Ti(5f) Cations}

We also considered the $\mathrm{Ti}(5 \mathrm{f})$ atoms adjacent to the vacancy as possible adsorption sites. Although we find two stable spin-symmetry states, the binding energies are small compared to those of $\mathrm{O}_{2}$ adsorbed at the vacancy. In the triplet state (see Table 4), the $\mathrm{O}_{2}$ molecule adsorbs by removing one of the excess electrons from the reduced surface, while the remaining excess electron diffuses towards the active $\operatorname{Ti}(5 \mathrm{f})$ cation site at which the molecule is adsorbed. This species is bound by an energy of $0.33 \mathrm{eV}$. In the quintet state, an $\mathrm{O}_{2}$ molecule is physisorbed at the active Ti(5f) cation site, and maintains its chemical identity. We find that the bond length of the adsorbed $\mathrm{O}_{2}$ molecule, $1.191 \AA$, is essentially identical to the bond length in the gas phase, $1.197 \AA$. As can be seen from the difference in Mulliken charge, $\delta=-0.037$, between the two oxygen atoms in $\mathrm{O}_{2}$, the molecule is only slightly polarized towards the adsorption site.
Table 4. Summary of the results from calculations of $\mathrm{O}_{2}$ adsorbed on the $\mathrm{Ti}(5 \mathrm{f})$ cations adjacent to the vacancy, from ref 15. The superscript $a$ refers to the atom of the O molecule oriented towards the surface, the superscript $b$ to the atom further from the surface, the superscript $c$ to the $\operatorname{Ti}(5 \mathrm{f})$ cations on which $\mathrm{O}_{2}$ adsorb, and the superscript $d$ to the reduced Ti cation localized in the central layer

\begin{tabular}{lcc}
\hline parameter & triplet state & quintet state \\
\hline Binding energy $(\mathrm{eV})$ & 0.33 & 0.14 \\
Length of $\mathrm{O}_{2}$ bond $(\AA)$ & 1.329 & 1.191 \\
Spin density for $\mathrm{O}_{\text {prox }}{ }^{a}$ & 0.046 & 0.931 \\
Spin density for $\mathrm{O}_{\text {dist }}{ }^{b}$ & 0.949 & 1.064 \\
Spin density for $\mathrm{Ti}^{c}$ & 0.993 & 1.007 \\
Spin density for $\mathrm{Ti}^{d}$ & $\ldots$ & 0.991 \\
Mulliken charge for $\mathrm{O}_{\text {prox }}{ }^{a}$ & -0.880 & -0.077 \\
Mulliken charge for $\mathrm{O}_{\text {dist }}{ }^{b}$ & -0.058 & 0.040 \\
Electron transfer for $\mathrm{Ti}^{c}$ & -0.326 & -0.468 \\
Electron transfer for $\mathrm{Ti}^{d}$ & $\ldots$ & -0.468 \\
Distance of $\mathrm{O}_{\text {prox }}{ }^{a}$ to the surface & 1.808 & 1.865 \\
Distance of $\mathrm{O}_{\text {bridging }}$ to the surface & 1.103 & 1.114 \\
\hline
\end{tabular}

\subsubsection{Saturated Conditions: Adsorption of Three $\mathrm{O}_{2}$} Molecules

With the $(2 \times 3)$ unit cell we also modeled the maximum number of molecules that can be adsorbed per vacancy site. We find, in agreement with experiment, that the system in which three molecules are adsorbed is stable. The optimized structure for this system is depicted in Fig. 3, and the most significant results are presented in Table 5. The physical picture revealed by the calculations is that one $\mathrm{O}_{2}$ molecule reoxidizes the reduced surface and becomes $\mathrm{O}_{2}^{2-}$, while the other two $\mathrm{O}_{2}$ molecules, physisorbed on $\mathrm{Ti}(5 \mathrm{f})$ sites, maintain their identities as neutral molecules and experience a marked polarization $\left(\delta^{+,-}=0.21\right)$ towards the Ti center. This polarization is an indication that the bonding is electrostatic in nature. The spin density and Mulliken charge of the entire system, previously localized on Ti centers, is transfered to the $\mathrm{O}_{2}$ molecules that adsorb on the Ti(5f) sites, and to the $\mathrm{O}_{2}$ molecule adsorbed on the vacancy site, respectively.

Note in Table 5 that the charge on the peroxo $\mathrm{O}_{2}^{2-}$ species, -1.633 , is polarized towards the $\mathrm{O}$ atom closer to the surface ( -1.279 vs. -0.354$)$, which, in turn, has an overlap population with the $\mathrm{Ti}(4 \mathrm{f})$ center $(0.068)$ very near to that of the bridging oxygen atom at the same $\mathrm{Ti}$ site (0.077). The displacements of the ions close to the vacancy are very similar to those of the defect-free surface. For example, the two adjacent Ti atoms reverse the directions of their relaxations along the line normal to the surface from their positions in the reduced 


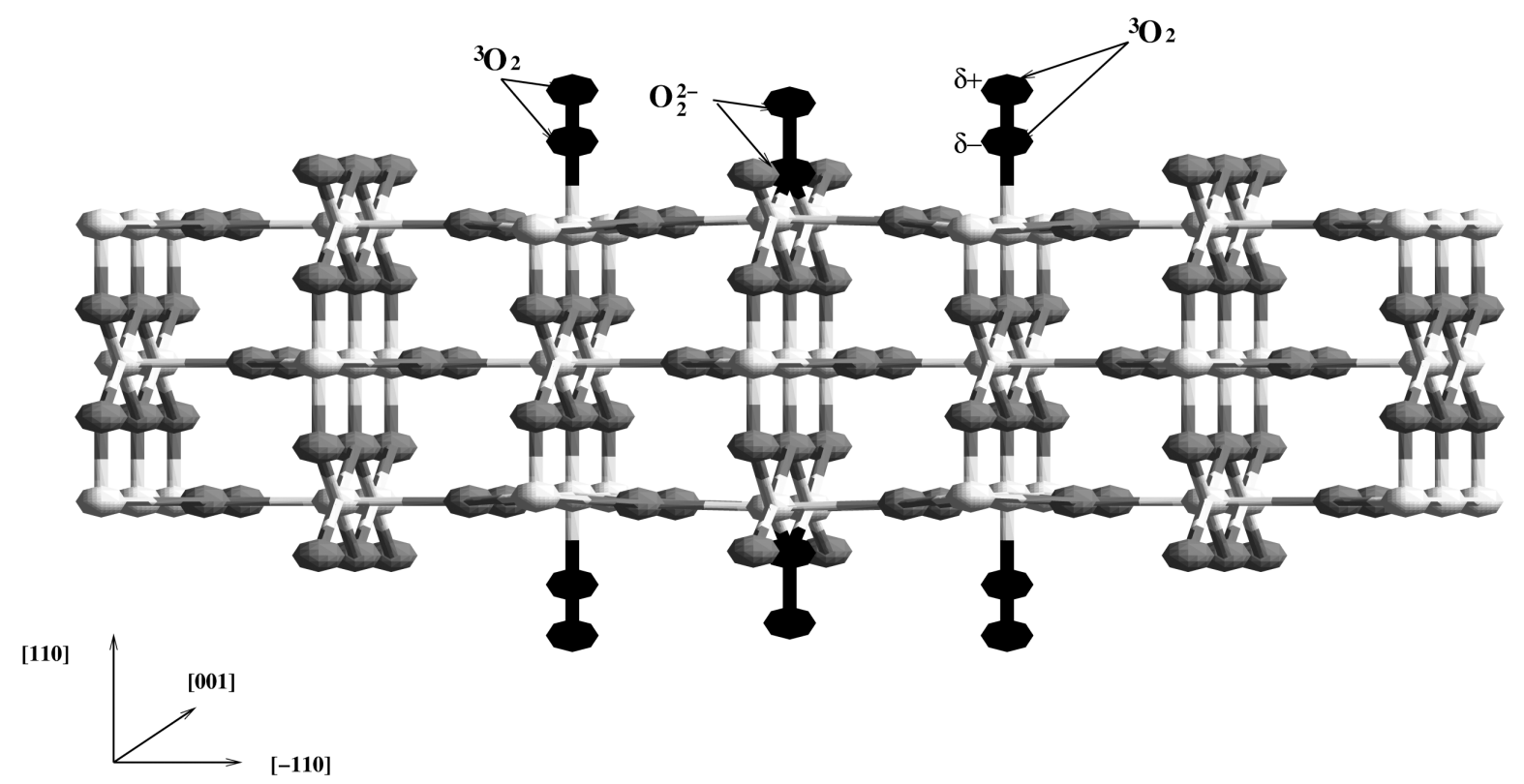

Fig. 3. Side view of the relaxed geometries calculated with three $\mathrm{O}_{2}$ molecules adsorbed on the reconstructed $2 \times 3 \mathrm{TiO}(110)$ surface. One of the $\mathrm{O}_{2}$ molecules is adsorbed at the anion defect site in a perpendicular orientation, the other $\mathrm{O}_{2}$ molecules are adsorbed on the Ti fivefold coordinated site, also in a perpendicular approach. Gray and white spheres indicate oxygen and titanium ions, respectively, and black spheres indicate the oxygen atoms of the adsorbed $\mathrm{O}_{2}$ molecules. The results are from ref 16; reprinted with permission from Elsevier.

surface. At the same time, due to the adsorption of $\mathrm{O}_{2}$ on $\mathrm{Ti}(5 \mathrm{f})$ sites, a partial increase of the coordination of $\mathrm{Ti}(5 \mathrm{f})$ occurs. As a consequence, the movement into the surface is less pronounced $(0.092$ vs. $0.15 \AA)$. Since the main driving force in the relaxation is to compensate for the coordination of under-coordinated Ti ions, this result is easily understood. All of the evidence from these results indicates that the reoxidation of the reduced surface is complete after adsorption of three $\mathrm{O}_{2}$ molecules.

We have considered additional structures and spin symmetries for the adsorption of three molecules. For example, a configuration in which the two $\mathrm{O}_{2}$ molecules adsorbed on $\operatorname{Ti}(5 \mathrm{f})$ are tilted by $45^{\circ}$ with respect to the [110] directions and pointed in opposite directions was calculated to be $0.20 \mathrm{eV}$ higher in energy than the structure in which the two $\mathrm{O}_{2}$ molecules are in a perpendicular orientation. This result corroborates the interpretation that the adsorption of these molecules is mainly due to polarization effects.

\subsection{Overview of Results from Periodic Calculations}

The main conclusions from the calculations we have reported in this section are the following:

(a) When the reduced surface adsorbs one $\mathrm{O}_{2}$ molecule per defect site, the most stable species is assigned as $\mathrm{O}_{2}^{-}$chemisorbed at the vacancy in a perpendicular orientation to the surface, in a triplet spin-symmetry state. Part of the excess electron density of the reduced surface is retained in the subsurface region and the surface remains active for the adsorption of additional $\mathrm{O}_{2}$ molecules. The similarity between the results obtained with the reduced surface modeled with $(2 \times 1)$ and $(2 \times 3)$ supercells indicates that in this case the adsorption mechanism is highly localized.

(b) That triplet state is close in energy to a singlet state $(\Delta E=0.49 \mathrm{eV})$ in which the adsorbed molecule is best characterized as $\mathrm{O}_{2}^{2-}$. The formation of this species requires the migration of excess electronic density from $\mathrm{Ti}$ atoms in the subsurface towards the vacancy. This process is not well-localized, and as a consequence the adsorption energies calculated with $2 \times 1$ and $2 \times 3$ supercells are rather different (1.96 vs. $3.12 \mathrm{eV})$.

(c) Adsorption on the Ti(5f) cation sites is stable. However, the calculated adsorption energies are much smaller than those calculated for molecules chemisorbed at the vacancy.

(d) In saturated conditions, three molecules per vacancy can adsorb. The molecule on the defect site is assigned as a peroxo $\mathrm{O}_{2}^{2-}$ species chemisorbed at the vacancy. The other two molecules are physisorbed, 
Table 5. Summary of results from calculations of three $\mathrm{O}_{2}$ molecules $\left(3 \mathrm{O}_{2} / \mathrm{TiO}_{2}\right)$ adsorbed on the reduced $\mathrm{TiO}_{2}(110)$ surface modeled by means of a $(2 \times 3)$ supercell, from ref 16 . Data in parentheses are from the calculations on the reduced surface modeled by means of a $2 \times 1$ supercell, from ref 16

\begin{tabular}{|c|c|}
\hline parameter & $3 \mathrm{O}_{2} / \mathrm{TiO}_{2}$ \\
\hline Binding energy $(\mathrm{eV})$ & $\begin{array}{c}1.32 \\
(1.24)\end{array}$ \\
\hline Length of $\mathrm{O}_{2}{ }^{a}$ bond $(\AA)$ & $\begin{array}{c}1.596 \\
(1.537)\end{array}$ \\
\hline Spin density for $\mathrm{O}_{\text {prox }}^{b}$ & $\begin{array}{c}0.0 \\
(0.0)\end{array}$ \\
\hline Spin density for $\mathrm{O}_{\text {dist }}{ }^{c}$ & $\begin{array}{c}0.0 \\
(0.0)\end{array}$ \\
\hline Mulliken charge for $\mathrm{O}_{\text {prox }}{ }^{b}$ & $\begin{array}{l}-1.279 \\
(-1.284)\end{array}$ \\
\hline Mulliken charge for $\mathrm{O}_{\text {dist }}{ }^{c}$ & $\begin{array}{l}-0.354 \\
(-0.336)\end{array}$ \\
\hline Length of $\mathrm{O}_{2}{ }^{d}$ bond $(\AA)$ & $\begin{array}{c}1.185 \\
(1.194)\end{array}$ \\
\hline Mulliken charge for $\mathrm{O}_{\text {prox }}{ }^{e}$ & $\begin{array}{c}-0.224 \\
(-0.350)\end{array}$ \\
\hline Mulliken charge for $\mathrm{O}_{\text {dist }}{ }^{f}$ & $\begin{array}{c}0.203 \\
(0.327)\end{array}$ \\
\hline Spin density for $\mathrm{O}_{p r o x}^{e}$ & $\begin{array}{c}0.747 \\
(0.597)\end{array}$ \\
\hline Spin density for $\mathrm{O}_{\text {dist }}{ }^{f}$ & $\begin{array}{c}1.251 \\
(1.398)\end{array}$ \\
\hline Distance of $\mathrm{O}_{\text {prox }}{ }^{b}$ to $\mathrm{Ti}(4 \mathrm{f})$ & $\begin{array}{c}1.875 \\
(1.900)\end{array}$ \\
\hline Distance of $\mathrm{O}_{\text {prox }}{ }^{e}$ to $\mathrm{Ti}(5 \mathrm{f})$ & $\begin{array}{c}2.036 \\
(2.012)\end{array}$ \\
\hline Distance of $\mathrm{O}_{\text {bridging }}$ to $\mathrm{Ti}(4 \mathrm{f})$ & $\begin{array}{c}1.792 \\
(1.796)\end{array}$ \\
\hline
\end{tabular}

${ }^{a} \mathrm{O}_{2}$ molecule adsorbed at anion defect site. ${ }^{b}$ Atom of oxygen oriented towards the surface for $\mathrm{O}_{2}$ adsorbed at anion defect site. ${ }^{c}$ Atom of oxygen oriented away from the surface for $\mathrm{O}_{2}$ adsorbed at anion defect site. ${ }^{d} \mathrm{O}$ molecules adsorbed on cation $\mathrm{Ti}(5 \mathrm{f})$ sites. ${ }^{e}$ Atom of oxygen oriented away from the surface for $\mathrm{O}_{2}$ adsorbed on cation $\mathrm{Ti}(5 \mathrm{f})$ site. ${ }^{f}$ Atom of oxygen oriented away from the surface for $\mathrm{O}_{2}$ adsorbed on cation $\mathrm{Ti}(5 \mathrm{f})$ site.

remain neutral, and are polarized towards the $\operatorname{Ti}(5 f)$ centers.

(e) Complete reoxidation of the surface requires the migration of excess electrons from Ti atoms of the subsurface towards the surface.

\subsection{Relationship to Experimental Observations}

The results of the extended calculations, in which the reduced surface is modeled with a percentage of vacancies $(16 \%)$ similar to that in the experiments $(8 \%)$, can be used to interpret the results of Henderson et al. ${ }^{7}$ First, the experiments detect temperature-programmed desorption peaks associated with chemisorption (at $410 \mathrm{~K}$ ) as well as physisorption (at $145 \mathrm{~K}$ ). As discussed above, we have also found stable species with binding energies corresponding to chemisorption and physisorption. Second, the experiments find spectroscopic (HREELS and EELS) evidence that the chemisorption peak is due to the presence of $\mathrm{O}_{2}^{-}$. According to our calculations, this species is the most stable one on the surface. Also, our results reveal that the oxygen atoms of the $\mathrm{O}_{2}^{-}$species are inequivalent, which would allow them to possess an allowed infrared $\mathrm{O}-\mathrm{O}$ stretching signal in the HREELS spectrum. ${ }^{7}$ Finally, in close agreement with the best ionpacking model proposed experimentally, ${ }^{7}$ we show that in saturated conditions three molecules are adsorbed per vacancy. In the most stable arrangement, one molecule is chemisorbed on the defect site and two are physisorbed on adjacent $\mathrm{Ti}(5 \mathrm{f})$ cation sites.

Interpreting the photodesorption experiments of Yates and coworkers ${ }^{10}$ requires information about the excited states and couplings in the molecule-surface system. Since, according to Perkins and Henderson, ${ }^{9}$ the $410 \mathrm{~K}$ TMP peak stems from the $\alpha$ species detected by Yates, experimentally characterized as $\mathrm{O}_{2}^{-}$adsorbed on the surface, it seems logical to assume that this species is responsible for the observed $\mathrm{O}_{2}$ photodesorption at lower temperature $(100 \mathrm{~K})$. Taking advantage of the localized character of the adsorption process for this species, in which the charge transfer between a single $\mathrm{O}_{2}$ and localized $3 \mathrm{~d}$ levels of Ti centers is the main physical effect, we resort to a cluster model. This is the subject of the following section. With regard to the conversion of the $\alpha$ species to the $\beta$ species at $250 \mathrm{~K}$, and the characterization of the latter species, we can speculate that temperature-dependent spin-flip of the vacancy electrons might be responsible for the irreversible character of the transformation, and that the $\beta$ species may correspond to a different spin-symmetry state of the $\mathrm{O} / \mathrm{TiO}_{2-x}$ system. Another possibility is that the $\beta$ species corresponds to the triplet state in which $\mathrm{O}_{2}$ bounds on the top of the Ti(5f) site (see Table 4).

Finally, it should be noted that in the time-resolved STM measurements of Schaub et al. ${ }^{12}$ and Wahlström et al., ${ }^{11}$ only $\mathrm{O}_{2}$ molecules adsorbed on the $\mathrm{Ti}(5 \mathrm{f})$ cations were detected. The higher adsorption temperature $\left(\mathrm{T}_{a} \sim 250 \mathrm{~K}\right)$ used in these experiments might be responsible for the apparent discrepancy with previous experimental results. In fact, as mentioned above, Henderson et al. ${ }^{7,9}$ found that $\mathrm{O}_{2}$ adsorbs at the vacancy in molecular form only if $\mathrm{T}_{a}<150 \mathrm{~K}$. At higher temperatures, $\mathrm{O}_{2}$ dissociates. Also, in the STM experiments, ${ }^{12}$ the density of adsorbed $\mathrm{O}_{2}$ molecules is much lower than the density of surface vacancy sites, while in the controlled 
PD and TPD experiments the $\mathrm{O}_{2}$ coverage is of the same order as the vacancy density. A theoretical investigation of the chemistry of $\mathrm{O}_{2}$ on the defect surface under the experimental STM conditions would require extremely large supercells. In addition, predicting the mechanism would require a complete map of the ground-state potential energy surfaces and calculations of the barrier heights for the molecular and dissociative desorption channels, as well as $\mathrm{O}_{2}$ diffusion from a vacancy onto a Ti(5f) cation. The STM experiments have been interpreted recently by $\mathrm{Wu}$ et al..$^{39}$ and Rasmussen et al. ${ }^{40}$ using the Car-Parrinello method with plane-wave basis sets, and gradient-corrected DFT calculations, respectively. Both DFT calculations showed the singlet $\mathrm{O}_{2}^{2-}$ state to be below (about $1 \mathrm{eV}$ in ref 39) the triplet $\mathrm{O}_{2}^{1-}$ state. In contrast (see above) we find that the singlet species is less stable than the triplet, with an energy difference rather sensitive to the size of the supercell. Although it is generally more difficult to describe highspin density states than singlet states at the DFT level (see, for example, ref 41 and references therein), and the computational method and supercell models are different in these studies, the reason for the discrepancy is not obvious. Future work on this point would require a deeper study using various DFT, hybrid HF/DFT, and semiempirical approaches.

\section{EXCITED STATE AND NONADIABATIC COUPLING CALCULATION: A CLUSTER MODEL}

Studies of photodesorption require information about electronic excited states and the couplings among them. Obtaining such information from the periodic slab model is not feasible computationally. However, as described above, we find that the adsorption mechanism for molecular oxygen on $\mathrm{TiO}_{2}$, in the most stable spinsymmetry state and geometrical arrangement, has a strongly localized character. This allows us to use a cluster model to describe the relevant regions of the surface.

We model the most stable $\mathrm{O}_{2} / \mathrm{TiO}_{2-x}$ surface as a hydrogen-saturated cluster of stoichiometry $\mathrm{Ti}_{2} \mathrm{O}_{9} \mathrm{H}_{11}$, in which the number of protons is chosen such that the entire cluster has an electronic charge of $-1 .{ }^{42}$ Previous studies have shown that hydrogen-saturated clusters provide an accurate description of the reduced $\mathrm{TiO}_{2}$ (110) surface $^{43}$ and the desorption of small molecules ${ }^{44}$ from a clean $\mathrm{TiO}_{2}$ (110) surface. The success of the cluster model may be partly due to the fact that the Ti-O bond in $\mathrm{TiO}_{2}$ has both covalent and ionic character. ${ }^{43}$ The model chosen in this work is illustrated in Fig. 4. A single reduced electron is implied in this structure since, according to our extended calculations, when a single $\mathrm{O}_{2}$ molecule is adsorbed per defect site, a single excess electron from the reduced surface is transfered to the $\mathrm{O}_{2}$ molecule.

In Table 6 the results from the extended calculations are compared to those from the cluster at a restricted open-shell Hartree-Fock (ROHF) level. We can see that both energetic and structural data are in good agreement, even though the Mulliken charges on the adsorbate atoms are slightly different. However, the basis sets used in the two calculations were different, ${ }^{42}$ therefore Mulliken populations can be compared only on a qualitative basis. Note that the method used to calculate the surfaces and couplings presented below uses a much

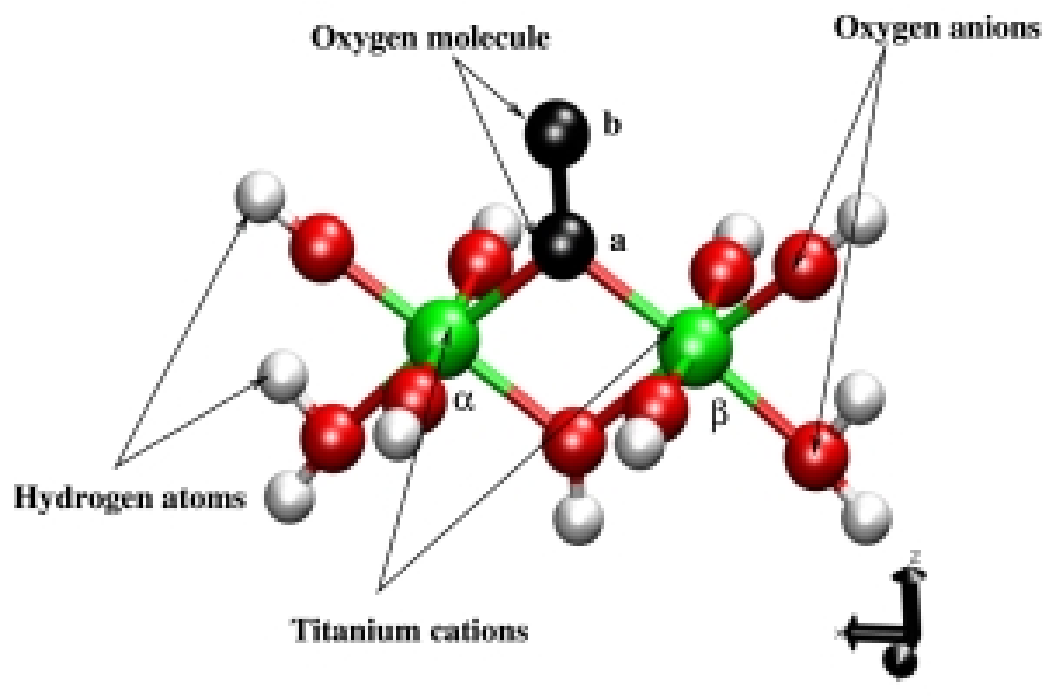

Fig. 4. Hydrogen-saturated cluster used to model the $\mathrm{O}_{2} / \mathrm{TiO}_{2-x}$ system, del $\mathrm{O}_{2} / \mathrm{TiO}_{2}: \mathrm{Ti}_{2} \mathrm{O}_{9} \mathrm{H}_{11}$. Reprinted from ref 42 , with permission from the publisher. 
Table 6. Comparison of HF results from slab ${ }^{15,16}$ and cluster model calculations of one $\mathrm{O}_{2}$ molecule adsorbed on the reduced $\mathrm{TiO}_{2}(110)$ surface in the ground state, from ref 42 . The superscript $a$ refers to the atom of the $\mathrm{O}_{2}$ molecule oriented towards the surface, and the superscript $b$ to the atom further from the surface

\begin{tabular}{lcc}
\hline parameter & $\begin{array}{c}\text { Slab } \\
\mathrm{O} / \mathrm{TiO}\end{array}$ & $\begin{array}{c}\text { Cluster } \\
\mathrm{O} / \mathrm{TiO}\end{array}$ \\
\hline Binding energy $(\mathrm{eV})$ & 3.61 & 3.60 \\
Length of $\mathrm{O}_{2}{ }^{a}$ bond $(\AA)$ & 1.373 & 1.398 \\
Spin density for $\mathrm{O}_{\text {prox }}{ }^{a}$ & -0.022 & 0.064 \\
Spin density for $\mathrm{O}_{\text {dist }}{ }^{b}$ & 1.026 & 0.920 \\
Mulliken charge for $\mathrm{O}_{\text {prox }}{ }^{a}$ & -0.959 & -0.735 \\
Mulliken charge for $\mathrm{O}_{\text {dist }}{ }^{b}$ & 0.071 & 0.002 \\
Distance of $\mathrm{O}_{\text {prox }}{ }^{a}$ to Ti(4f) & 2.031 & 2.006 \\
Distance of $\mathrm{O}_{\text {bridging }}$ to Ti(4f) & 1.800 & - \\
\hline
\end{tabular}

higher level of correlation than Hartree-Fock. The results in Table 6 are presented for consistency.

We find that an efficient and accurate way to calculate the potential surfaces is to perform separate CASSCF optimizations for each electronic state. The CASSCF method accounts for the most important nondynamic correlation effects. The remaining dynamic correlation is evaluated using an internally contracted MRCI approach, ${ }^{45}$ including the Davidson correction, $\mathrm{MRCI}+\mathrm{Q}{ }^{46}$

The potential curves obtained with this method are displayed in Fig. 5 as a function of the distance, denoted $Z$, between the $\mathrm{O}_{2}$ center-of-mass and the Ti-surface plane. The $\mathrm{O}_{2}$ bond length has been fixed at its equilibrium value. ${ }^{42}$ Note, in the figure, that the ground state,

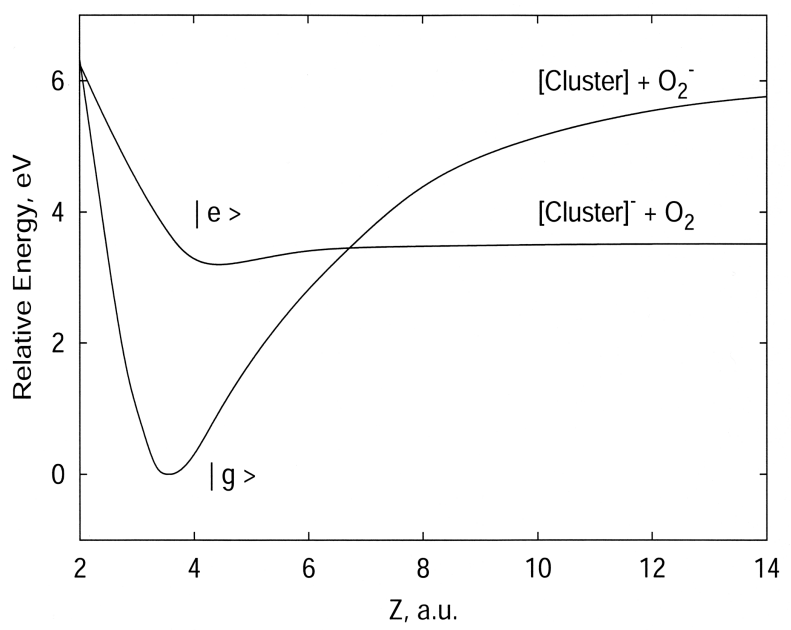

Fig. 5. States involved in the light-induced desorption of $\mathrm{O}_{2}$ from $\mathrm{TiO}_{2}$. The coordinate $\mathrm{Z}$ is defined as the distance between the $\mathrm{O}_{2}$ center of mass and the Ti-surface plane (labeled $\mathrm{Z}=0$ ). $|g\rangle\left(1^{1} A^{\prime}\right)$, and excited state, $|e\rangle\left(1^{1} A^{\prime}\right)$, have radically different electronic character. The ground state describes the strong Coulomb interaction $(5.85 \mathrm{eV})$ between a negatively charged adsorbate and the surface, and correlates asymptotically to [cluster] $+\mathrm{O}_{2}^{-}$. In contrast, the excited state corresponds to the interaction of a neutral molecule with the substrate (physisorption), and correlates to [cluster] ${ }^{-}+\mathrm{O}_{2}$. In the excited state the $\mathrm{O}_{2}$ molecule is polarized towards the cluster. This inductive attraction causes a small minimum in the interaction potential with a well-depth of about $0.31 \mathrm{eV}$.

Since the ground and excited states have such a different electronic character, the corresponding optimized orbitals for these two states are also very different. Therefore, methods using a common set of orbitals would require very large CI expansions to describe both states correctly and thus would be prohibitively expensive. We have checked that even with large active spaces, the common orbital CASSCF approach describes both states poorly, especially the relative energies. As a result, it is natural to use orbitals optimized separately for each state. To ensure continuous behavior of the electronic structure along $Z$, we adopted the following strategy. We start the calculation at large distances, where the neutral oxygen molecule (and ion) are well separated from the cluster. At this point, the nature of the two states is easily identified, and the orbitals are generated by restricted HF with appropriate occupation patterns. The correlated calculations that follow do not modify the structure of the wave function drastically (this is monitored using a Mulliken population analysis). Starting from this distance, we move inward, using the previous geometry as a starting guess, and ensuring that the orbitals are not modified strongly. This procedure allows us to preserve the character of the two states over the entire distance interval, and avoid mixing with the many excited states that are unavoidably present in the energy interval of interest. Application of this method produces states that are best described as diabatic states. The degree of nondiabaticity can be checked by calculating the corresponding derivative coupling (see below). It is clear, though, that these states will be neither exactly orthogonal nor exactly diagonalize the electronic Hamiltonian matrix.

To calculate the electronic coupling, $\langle 1|\hat{H}| 2\rangle$, and the overlap, $\langle 1 \mid 2\rangle$, for the CASSCF and ICMRCI wave functions built with different, non-orthogonal orbitals, ${ }^{47}$ we follow the method suggested by Malmqvist, ${ }^{48}$ using a transformation to bi-orthogonal orbitals. This procedure is accomplished by performing a Singular Value Decomposition (SVD) of the orbital overlap matrix,

$$
\mathbf{S}=\mathbf{U}^{\mathrm{T}} \mathbf{D V}
$$


where $\mathbf{U}$ and $\mathbf{V}$ are unitary, $\mathbf{T}$ is the transpose of $\mathbf{U}$, and D is a diagonal matrix. We then rotate left and right CI states using $\mathbf{U}$ and $\mathbf{V}$ as described by Malmqvist, and scale one set using $\mathbf{D}$, to express the $\mathrm{CI}$ coefficients in the new rotated orbitals. After this procedure, the corresponding left and right orbitals are orthogonal to each other (bi-orthogonal). When the left and right states are expressed in bi-orthogonal orbitals, standard techniques can be used to evaluate overlaps and matrix elements, with minor modifications due to the nonsymmetric character of the one- and two-electron integrals.

This approach was first implemented for CASSCF and RASSCF wave functions in the RASSI program of the MOLCAS quantum chemistry package. ${ }^{49}$ One advantage of using the MOLPRO program is that the ICMRCI program allows for a very accurate description of dynamic correlation. However, for ICMRCI wave functions the application of the bi-orthogonal transformation is quite complicated, as the full orbital set transformation destroys the special maximum-double-excitation structure of MRCI wave functions. These resulting wave functions are essentially the same size as full-CI size wave functions, which makes the evaluation of matrix elements much more demanding, and impossible in practice.

To solve this problem, Malmqvist ${ }^{48}$ showed that it is possible, using a non-unitary transformation of the special form,

$$
\mathrm{U}=\left(\begin{array}{ccc}
\mathrm{U}_{\mathrm{cc}} & \mathrm{U}_{\mathrm{ca}} & \mathrm{U}_{\mathrm{cs}} \\
0 & \mathrm{U}_{\mathrm{aa}} & \mathrm{U}_{\mathrm{as}} \\
0 & 0 & \mathrm{U}_{\mathrm{ss}}
\end{array}\right)
$$
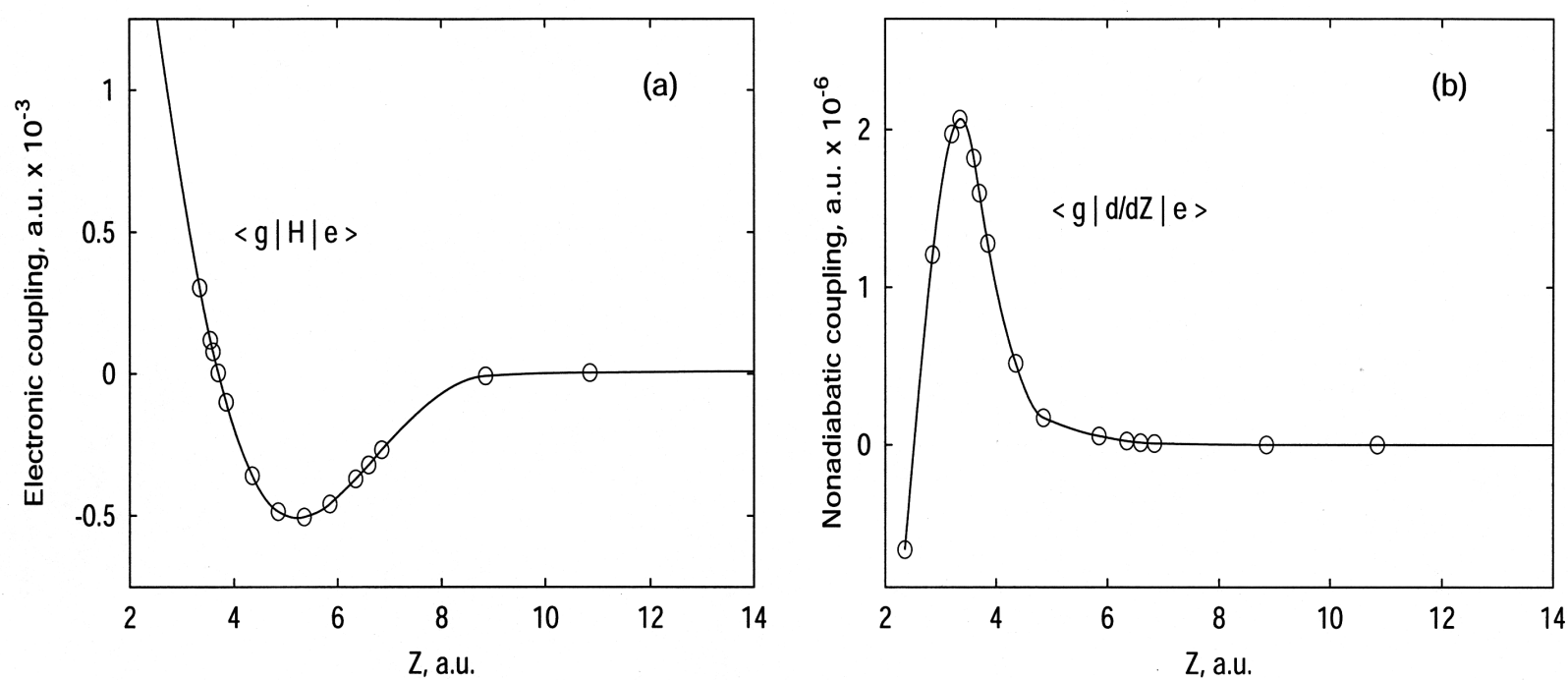

Fig. 6. Electronic coupling, $\left\langle\phi_{g}|\hat{H}| \phi_{e}\right\rangle$, (a), and derivative coupling, $\left\langle\phi_{g}\left|\frac{d}{d Z}\right| \phi_{e}\right\rangle$, (b), between the electronic states depicted in Fig. 5.

where indices $c, a, s$ refer to the core, active, and secondary orbitals, to preserve the MRCI structure. We have proven that the same procedure is valid for internally contracted MRCI wave functions, and have implemented the method within MOLPRO.

As mentioned above, the states obtained with different orbitals are non-orthogonal. For this system we found the non-orthogonality to be very small, reflecting the fact that the states preserve their (very different) ionic character. This small non-orthogonality is related to the incompleteness of the CASSCF and MRCI spaces, which produces a "spurious" small mixing between "true" diabatic states. To eliminate this mixing we apply a symmetric orthogonalization, with $\hat{S}^{-1 / 2}$, and recalculate the electronic coupling, $\langle 1|\hat{H}| 2\rangle$. After reorthogonalization, the states approximate much better the "true" diabatic states. In fact, the couplings calculated in this way prove to be quite stable, nearly invariant for CASSCF and ICMRCI wave functions obtained with different active spaces. ${ }^{47}$ This observation is an important confirmation of the validity of our approach.

To check the degree of diabaticity of the orthogonalized states, we estimate the magnitude of the derivative coupling (evaluated with a finite difference approach), $\frac{1}{\mu}\left\langle\phi_{g}\left|\frac{d}{d Z}\right| \phi_{e}\right\rangle \frac{d}{d Z}$ and compare them to the electronic coupling, $\left\langle\phi_{g}|\hat{H}| \phi_{e}\right\rangle$. The results of these calculations are presented in Fig. 6. Note that if the calculated potentials were adiabatic, the couplings would be very large at the avoided crossing point and decay rapidly to zero away from the crossing. As can be seen in the figure, the magnitude of the derivative coupling matrix element $\left\langle\phi_{g}\left|\frac{d}{d Z}\right| \phi_{e}\right\rangle$ is in fact quite small $\left(\sim 2 \times 10^{-5}\right.$ at most $)$, and

Israel Journal of Chemistry $\quad 45 \quad 2005$ 
not correlated to the position of the crossing. In fact, the maximum is centered in the Frank-Condon region. As we mentioned, these nonadiabatic derivative matrix elements enter the nuclear Hamiltonian as $\left\langle\phi_{g}\left|\frac{d}{d Z}\right| \phi_{e}\right\rangle$. Using harmonic oscillator wave functions, it is possible to estimate the order of magnitude of these matrix elements as $\sim \sqrt{\mu w / 2}$. Therefore, the total contribution from this term is of the order $\sqrt{w / 2 \mu}\left\langle\phi_{g}\left|\frac{d}{d Z}\right| \phi_{e}\right\rangle$. Here we used the fact that $\left\langle\phi_{g}\left|\frac{d}{d Z}\right| \phi_{e}\right\rangle$ is a slowly-varying function of $Z$ because the states are nearly diabatic. Using the $\mathrm{O}_{2}$ reduced mass and a typical vibrational frequency $\left(1000 \mathrm{~cm}^{-1}\right)$, the factor $\sqrt{\mu w / 2}$ is $\sim 2 \times 10^{-4}$, which implies a total contribution of order $4 \times 10^{-9}$. This is negligible compared to $\left\langle\phi_{g}|\hat{H}| \phi_{e}\right\rangle$. This estimate confirms that our approach does, in fact, produce nearly diabatic states coupled by a non-diagonal matrix element of the electronic Hamiltonian, $\left\langle\phi_{g}\left|\hat{H}_{e l}\right| \phi_{e}\right\rangle$. In the following, we will refer to this term as the diabatic coupling.

\section{LIGHT-INDUCED DESORPTION DYNAMICS}

In previous work we performed a theoretical study of the light desorption process by considering a direct optical excitation mechanism of the adsorbate-substrate bond..$^{42}$ This mechanism is justified given the magnitude of the corresponding dipole moment between the ground and the excited state $(\sim 0.01$ a.u. at the equilibrium adsorbate-surface distance). The initial state is given by the transition dipole operator acting on the first vibrational level of the ground state. We assume that the two surfaces are coupled by a Gaussian-shape laser pulse. The time evolution of the wave function is monitored by solving the time-dependent Schrödinger equation for the coupled-surface problem using a Lanczos procedure, ${ }^{50}$ combined with FFT techniques for numerical fast derivatives. We calculate the desorption probability as a function of the central frequency and compare the resulting desorption profile to the experimental data. The results from these calculations are shown in Fig. 7. The desorption probability has a nearly Gaussian form with its peak at the inflection point in the experimental data. The half-width at half-maximum, $\Gamma(\mathrm{HWHM})$, of the calculated profile can be related to the average kinetic energy gained by the desorbed $\mathrm{O}_{2}$ molecules. From $\Gamma$ (HWHM) we can estimate an average kinetic energy of $0.12 \mathrm{eV}$, which is in close agreement with the laser-induced desorption experiments from Möller et al. $(0.14 \mathrm{eV}) .^{13}$

The disagreement between theory and experiment above the excitation energy $\left(\mathrm{T}_{e}\right)$ may indicate that additional desorption mechanisms are operative for higher photon-energies. Based on the nature of the first excited state, we suspect that a hole-induced desorption mechanism is operative above the band gap of the reduced

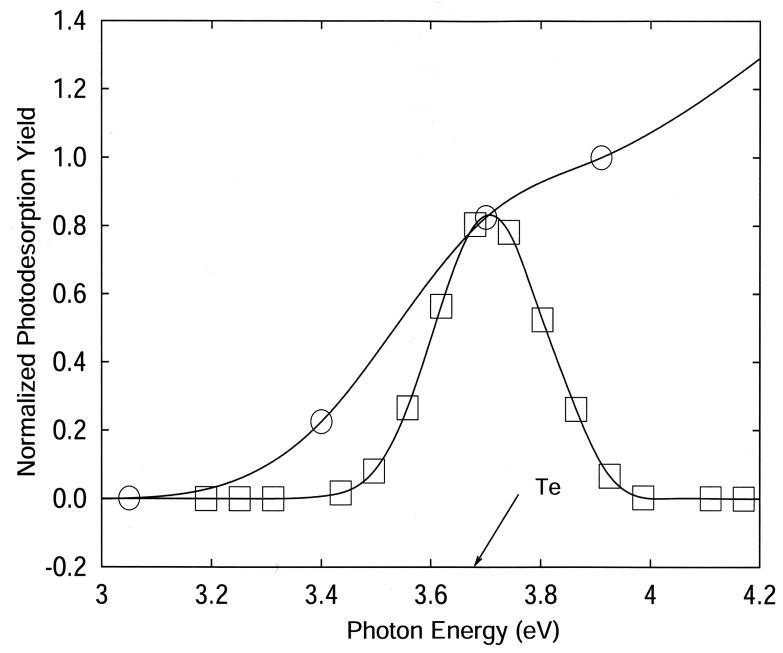

Fig. 7. Normalized desorption yield of $\mathrm{O}_{2}$ as a function of the central frequency of the laser pulse. The theoretical desorption probability (circles) has been normalized to the experimental photodesorption yield (squares) at the vertical excitation energy $\left(\mathrm{T}_{e}\right)$ between the ground and the first excited state, $\mathrm{T}_{e}$. The experimental data are from ref 10 . Reprinted from ref 42 , with permission from the publisher.

surface, and becomes dominant at energies above $\mathrm{T}_{e}$. It is also possible that excited vibrational modes of the adsorbed molecule may contribute to the experimental photodesorption profile.

The next step is to analyze the dependence of the desorption cross section on the nonadiabatic coupling between the ground and excited states. Our initial calculations, with a simple, Gaussian model for the couplings, showed a strong dependence on the parameters of the model. To quantify this effect, the calculations were repeated with ab initio coupling calculated with the method described above.

As a first step, we considered a one-dimensional model of the photodesorption dynamics. We calculated two coupled potential energy curves as a function of the distance of the center of the $\mathrm{O}_{2}$ molecule from the $\mathrm{TiO}_{2}$ surface. The bond length and the orientation of $\mathrm{O}_{2}$ are fixed at their equilibrium geometry, and then the dipole moment matrix element, d, connecting the two electronic state is calculated. This simplified model is not intended to be realistic, but it can be used as an estimate of the extent to which the electronic coupling must be included in the photodesorption dynamics.

In a first-order perturbative treatment for electric dipole transitions, the absorption from an initial bound state $\Phi_{g v}$ of the system to a dissociative continuum at energy $E$ is given by

$$
\left.\sigma(E) \propto \sum_{\beta}\left\langle\Phi_{g v}|\mathbf{d} \cdot \mathbf{e}| \Psi^{\beta, E}\right\rangle\right|^{2}
$$


In this equation, $\mathbf{e}$ is the polarization vector of the incident light (linear in the present case), and the transition dipole moment is directed along the $\mathrm{O}_{2}$-surface distance. The initial state is approximated by $\Phi_{g v} \approx \varphi_{v}(R)|g\rangle$, where $\varphi_{v}(R)$ is a vibrational eigenfunction on the $|g\rangle$ electronic state. The dissociative wave functions are expanded as

$$
\Psi^{\beta, E}=\varphi_{g}^{\beta, E}|g\rangle+\varphi_{e}^{\beta, E}|e\rangle \quad \beta=g, e
$$

where the $\varphi_{e, g}^{\beta, E}$ coefficients are obtained as solutions of the time-independent close-coupling equations

$$
\left\{-\frac{\hbar^{2}}{2 \mu} \frac{d^{2}}{d R^{2}}+E_{\beta}(R)-E\right\} \varphi_{\beta}^{\beta, E}(R)=V_{\beta, \alpha}(R) \varphi_{\alpha}^{\beta, E}(R)
$$

at energy $E$, with $\beta \neq \alpha, E_{\beta}$ is the diagonal diabatic energy, and $V_{\beta, \alpha}=\left\langle\phi_{g}|\hat{H}| \phi_{e}\right\rangle$ is the electronic coupling. The resolution of these equations is performed using the Fox-Numerov method. ${ }^{51}$ The dipole matrix elements, the $\left\langle\varphi_{v}|d| \varphi_{\alpha}^{\beta, E}\right\rangle$, are evaluated using a procedure described elsewhere. ${ }^{52}$

The total absorption spectrum obtained with this method is shown in Fig. 8a. The spectrum displays a broad envelope with narrow structures superimposed. To explain the spectrum, two limiting cases for the dynamics are considered, diabatic or adiabatic. In the diabatic limit, the initial wave packet in the $|e\rangle$ physisorption diabatic state dissociates directly, without experiencing the coupling with the $|g\rangle$ ionic diabatic state. In this case, the photodesorption dynamics is rapid, and the spectrum shows a broad unstructured band, as can be seen in Fig. 8b. This spectrum is in good agreement with the spectrum calculated previously using a time-dependent method. ${ }^{42}$

In the adiabatic limit, a set of vibrational quasi-bound states of the upper adiabatic surface are excited, giving rise to a set of nearly isolated, Lorentzian peaks, as shown in Fig. 8c. The narrow width of the peaks is due to the slow electronic predissociation induced by the nonadiabatic coupling terms. The two fragmentation mechanisms, the direct-diabatic and the indirect-adiabatic pathways, interfere with each other, giving rise to the non-Lorentzian, Beutler-Fano profiles ${ }^{53}$ in the total spectrum of Fig. 8a. The narrow resonances in the spectrum would require very long propagation times to resolve with a time-dependent method.

The "diabatic spectrum" in Fig. 8b has been calculated "exactly" in the absence of the electronic coupling. However, the spectrum in the adiabatic limit has been approximated using a first-order perturbative treatment. In this procedure, the adiabatic electronic functions, $|+\rangle$ and $|-\rangle$, are obtained from an analytic diagonalization of

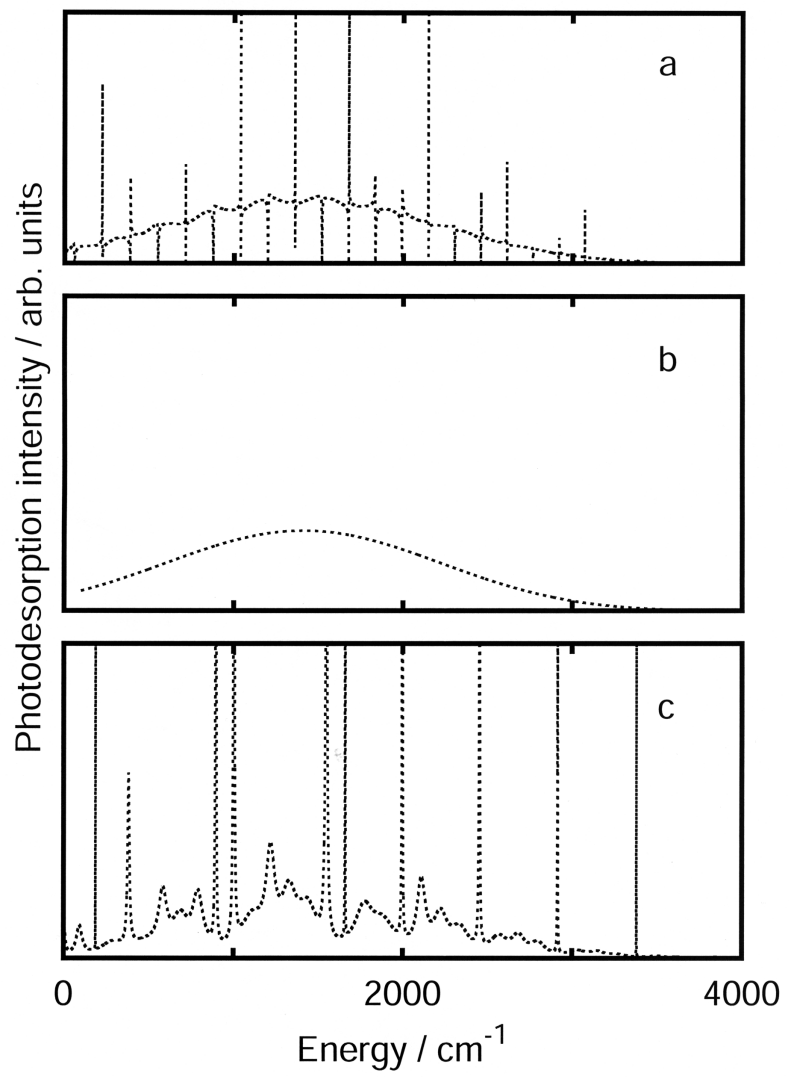

Fig. 8. Absorption spectrum for two electronic states in Fig. 5 with diabatic coupling (a), two states with no coupling (b), and two states with adiabatic coupling to first order in perturbation theory (c). The zero of energy corresponds to the asymptotic energy of the $|e\rangle$ diabatic state.

the $2 \times 2$ diabatic Hamiltonian matrix, such that

$$
\{|+\rangle,|-\rangle\}=\{|g\rangle,|e\rangle\}\left(\begin{array}{r}
\cos \alpha \sin \alpha \\
-\sin \alpha \cos \alpha
\end{array}\right)
$$

with $\tan \alpha=\left\{\Delta-\sqrt{\Delta^{2}+4 V_{g, e}^{2}}\right\} / 2$ and $\Delta=V_{g, g}-V_{e, e}$. The eigenvalues of the excited adiabatic curve, $E_{+, v}$, provide the energy of the resonances. The Lorentzian widths are obtained within a Golden-Rule approach as

$$
\Gamma=\pi\left|\left\langle\phi_{-, E}\left|\frac{\hbar^{2}}{\mu} \mathcal{D} \frac{d}{d R}\right| \phi_{+, v}\right\rangle\right|^{2} \text { with } E=E_{+, v}
$$

where $\phi_{+, v}$ are the bound eigenfunctions of the upper adiabatic curve, $\phi_{-, E}$ are the dissociative eigenfunctions on the ground adiabatic state, and the dynamical couplings are given by $\mathcal{D}=d \alpha / d R$. This formula predicts a perfect Lorentzian peak centered at the crossing point, $R_{c}=2.861$ a.u., with a half-width at half-maximum of 
0.0189 a.u. The values of $\Gamma$ vary strongly, between femtoseconds and several hundreds of picoseconds, as shown in Fig. 9. This strong dependence on the vibrational level arises from the magnitude of the wave functions near the crossing point, where the nonadiabatic coupling is localized. Higher perturbative corrections might also yield a smoother dependence on $\Gamma$ with energy.

The intensity of the absorption from the $v=0$ vibrational level of the ground electronic states to each vibrational state of the upper adiabatic state is given by

$$
I_{v}=\left.\left|\left\langle\phi_{-, v^{\prime}=0}\right| \cos ^{2} \alpha-\sin ^{2} \alpha\right) \mathbf{d}_{0}\left|\phi_{+, v}\right\rangle\right|^{2}
$$

This simplified adiabatic model allows a determination of the widths of each of the vibrational levels in the upper adiabatic potential that appears in the spectrum. The continuous background may also have some contribution from the broader resonances. The differences with the "exact" calculations can be attributed to the perturbative character of the adiabatic treatment.

At this level of approximation, the photodetachment dynamics shows two different mechanisms. One contribution is due to direct excitation of the upper, diabatic electronic state. The second, indirect mechanism is

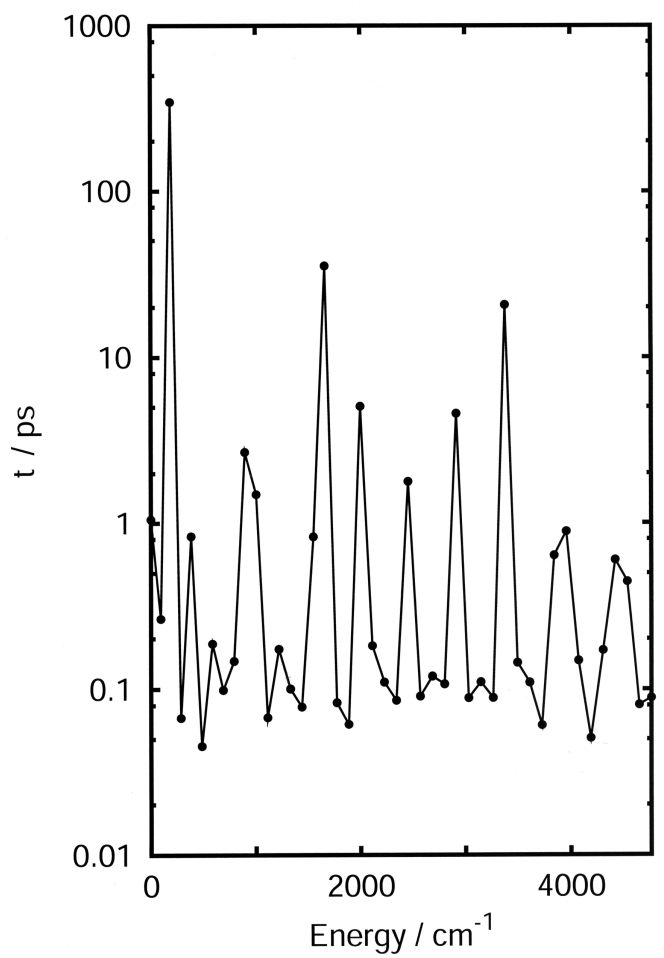

Fig. 9. Histogram of resonance lifetimes (in ps) of the bound states of the upper adiabatic electronic state in Fig. 5 obtained via a first-order, Golden-Rule approach. much slower, and results when the quasi-bound states supported by the upper adiabatic state predissociate via nonadiabatic couplings. The second mechanism is mediated by electronic coupling, and may have important implications on the complete dynamics.

In the one-dimensional model, the problem is analogous to the photodissociation of metal-halogen diatomic molecules, such as NaI. ${ }^{54}$ For NaI, the best description of the dynamics is within the adiabatic picture. The photon excitation creates an initial wave packet as a superposition of many vibrational states of the upper adiabatic state. The superposition state, or wave packet, oscillates in the bound portion of the potential well. Each time the wave packet reaches the crossing region, some of the amplitude is transferred to the ground electronic state. Time-independent methods. However, time-dependent methods, in which a spectroscopic investigation of the excited wave packet in the crossing region is performed with ultrashort laser pulses, allow an analysis of the transition state dynamics.

For the $\mathrm{O}_{2}$ on $\mathrm{TiO}_{2}$ problem, studies similar to those in NaI could, hypothetically, be performed. One difficulty, though, is that there are many additional degrees of freedom that might influence the dynamics. At low temperatures, diffusion can be neglected, so translational degrees of freedom can be neglected, as in the present approach. Since $\mathrm{O}_{2}$ occupies a vacancy in the $\mathrm{O}_{2} / \mathrm{TiO}_{2-x}$ complex, the bending motion of $\mathrm{O}_{2}$ relative to the surface is highly constrained, and can be neglected without loss of accuracy. At first glance, then, the most important degree of freedom required for a more realistic simulation of the photodetachment dynamics is the vibrational motion of $\mathrm{O}_{2}$. Including this degree of freedom would allow two competing fragmentation pathways to appear, the $\mathrm{O}_{2}$ photodetachment, and the fragmentation of $\mathrm{O}_{2}$ on the surface. Varying the parameters of the excitation might control, to some extent, which mechanism is dominant. In addition, the presence of long-lived resonances may lead to energy relaxation processes between the two mechanisms. This might enhance, for example, the fragmentation of $\mathrm{O}_{2}$, which would provide a control mechanism. Work along these lines, and a generalization of the work to two dimensions, is underway.

\section{CONCLUDING REMARKS AND FUTURE PROSPECTS}

In the present work we have developed a model to describe the adsorption and photo-induced desorption of $\mathrm{O}_{2}$ on a reduced $\mathrm{TiO}_{2}$ (110) surface. This model is able to explain the main features of recent experiments of Henderson and coworkers. ${ }^{79}$ At low-photon energies, 
our results are consistent with the controlled PD experimental results of Yates et al. ${ }^{10}$ We have also extended the model to include a full ab initio treatment of the nonadiabatic electronic interactions and quasi-diabatic potential energy curves and couplings. The resulting effects on the dynamics are of direct relevance to current and future experiments.

The main novelty of the present treatment consists of the extension of the ICMRCI method ${ }^{45}$ to obtain quasidiabatic states and couplings, corresponding to ionic substrate/adsorbate interactions. This approach was developed initially for an accurate treatment of molecular systems (see, for example, ref 55) and is based on the use of two separate sets of molecular orbitals to describe each of the electronic states. As far as we know, this is the only quantitative method applicable to such systems due to their large size, huge number of excited states describing excitations within the substrate, and interacting with the two ionic states of interest.

The photodesorption model presented in this work, although highly informative and qualitatively correct, is limited in some fundamental aspects. Considerable theoretical effort is required to understand this system in detail. Future work will include the generalization of the dynamics to higher-dimension models to study the competition between the desorption and dissociation channels of fragmentation. In particular, including the $\mathrm{O}_{2}$ vibrational mode is straightforward. In addition, we plan to study the role of resonances in determining which fragmentation channel dominates the dynamics. The presence of resonances may provide a mechanism for quantum control of the photodesorption process.

Another chemisorbed species that would be interesting to consider is the singlet species in which the absorbed molecule is characterized as $\mathrm{O}_{2}^{2-}$. This singlet peroxide state is close in energy to the triplet superoxide state we have studied in this work. For the singlet species, however, a larger cluster must be designed to model the charge migration from the subsurface to the vacancy, where it is captured by the neutral molecule approaching the surface. Also, as mentioned above, this species is a clear precursor to dissociative filling of the vacancies, and a realistic desorption model should include the $\mathrm{O}_{2}$ degree of freedom and, more likely, a dissociative state in which one oxygen atom fills the vacancy and the other oxygen atom adsorbs on the neighbor Ti(5f) site. Another aspect that might be considered is the scattering of $\mathrm{O}_{2}$ on the reduced surface, which might be strongly affected by the nonadiabatic coupling between the ground $\left(\mathrm{O}_{2}^{-}\right.$-surface $)$and the excited state $\left(\mathrm{O}_{2}\right.$-surface). In this regard, the recent work on molecular oxygen on the $\mathrm{Al}(111)$ surface (see ref 41) is particularly reassuring.
Acknowledgments. Many fruitful discussions with P. Palmieri, C. Pisani, N. Harrison, S.B. Trickey, D. Micha, H.J. Monkhorst, J.T. Yates, Jr., A. Perera, S. Fau, and P. Villareal are acknowledged gratefully. This work was partially supported by the US Department of Energy through grant DE-FG02-02ER45995, the DGICYT Spanish grant BFM 2001-2179, the TMR network HPRN-CT-1999-00005, and CINECA (HPRI-CT-1999-00048). M.P. de L-.C acknowledges financial support from the "Ramoñy Cajul" Programme.

\section{REFERENCES AND NOTES}

(1) Diebold, U. Surf. Sci. Phys. Rep. 2003, 48, 53-229.

(2) Fox, M.A.; Dulay, M.T. Chem. Rev. 1993, 93, 341-357.

(3) (a) Henrich, V.E.; Cox, P.A. The Surface Science of Metal Oxides; Cambridge University Press: Cambridge, 1993. (b) Fujishima, A.; Honda, K. Nature 1972, 238, 37-38. (c) Ikeda, K.; Sakai, H.; Baba, R.; Hashimoto, K.; Fujishima, A. J. Phys. Chem. B 1997, 101, 2617-2620.

(4) Linsebigler, A.L.; Lu, G.Q.; Yates, J.T.Jr. Chem. Rev. 1995, 95, 735-758.

(5) Fujishima, A.; Rao, T.N.; Tryk, D.A. Electrochim. Acta 2000, 45, 4683-4690.

(6) Heller, A. Acc. Chem. Res. 1995, 28, 503-508.

(7) (a) Henderson, M.A.; Epling, W.S.; Perkins, C.L.; Peden, C.H.F.; Diebold, U. J. Phys. Chem. B 1999, 103, 53285337. (b) Henderson, M.A.; Epling, W.S.; Peden, C.H.F.; Perkins, C.L. J. Phys. Chem. B 2003, 107, 534-545.

(8) Mills, A.; Davies, R.H.; Worsley, D. Chem. Soc. Rev. 1993, 22, 417-425.

(9) Perkins, C.L.; Henderson, M.A. J. Phys. Chem. B 2001, 105, 3856-3863.

(10) (a) Lu, G.Q.; Linsebigler, A.; Yates, J.T.Jr. J. Chem. Phys. 1995, 102, 3005-3008. (b) Lu, G.Q.; Linsebigler, A.; Yates, J.T.Jr. J. Chem. Phys. 1995, 102, 4657-4662. (c) Rusu, C.N.; Yates, J.T.Jr. Langmuir 1997, 13, 4311-4316.

(11) Wahlström, E.; Vestergaard, E.K.; Schaub, R.; Ronnau, A.; Vestergaard, M., Laegsgaards, E.; Stensgaard, I.; Besenbacher, F. Science 2004, 303, 511-513.

(12) Schaub, R.; Wahlström; E., Ronnau. A.; Laegsgaards, E.; Stensgaard, I.; Besenbacher, F. Science 2003, 299, 377-379.

(13) Möller, P.J.; Lazneva, E.F.; Komolov, A.S.; Komolov, A.K. Surf. Sci. 1998, 395, 82-87.

(14) Shu, C.; Sukumar, N.; Ursenbach, C.P. J. Chem. Phys. 1999, 110, 10539-10544.

(15) de Lara-Castells, M.P.; Krause, J.L. J. Chem. Phys. 2001, 115, 4798-4810.

(16) de Lara-Castells, M.P.; Krause, J.L. Chem. Phys. Lett. 2002, 354, 483-490.

(17) (a) Wolf, M.; Hasselbrink, E.; Ertl, G.; Zhu, X.Y.; White, J.M. Surf. Sci. 1991, 248, L235-L239. (b) Peremans, A.; Fukutani, K.; Mase, K.; Murata, Y.Phys. Rev. B 1993, 47, 4135-4138. (c) Zhu, X.Y.; White, J.M.; Junker, K.H. Chem. Phys. Lett. 1994, 226, 121126. (d) So, S.K.; Franchy, R.; Ho, W. J. Chem. Phys. 
1991, 95, 1385-1399. (e) Beauport, I.; Al-Shamery, K.; Freund, H.J. Chem. Phys. Lett. 1996, 256, 641-648.

(18) (a) Guo, H.; Chen, F. Faraday Discuss. 1997, 108, 309-326. (b) Guo, H.; Saalfrank, P.; Seideman, T. Prog. Surf. Sci. 1999, 62, 239-303. (c) Weik, F.; Demeijere, A.; Hasselbrink, E. J. Chem. Phys. 1993, 99, 682-694. (d) Kim, S.H.; Stair, P.C.; Weitz, E. Chem. Phys. Lett. 1999, 302, 511-516. (e) Micha, D.A.; Santana, A.; Salam, A. J. Chem. Phys. 2002, 116, 5173-5185.

(19) Brand, J.L.; George, S.M. Surf. Sci. 1986, 167, 341-362.

(20) (a) Nishi, N.; Shinohara, H.; Okuyama, T. J. Chem. Phys. 1984, 80, 3898-3910. (b) Harrison, I.; Polanyi, J.C.; Young, P.A. J. Chem. Phys. 1988, 89, 1475-1497. (c) Szulcsewski, G.J.; White, J.M.J. Vac. Sci. Technol. A 1997, 15, 1526-1530.

(21) (a) Zhu, X.Y.; Hatch, S.R.; Campion, A.; White, J.M. J. Chem. Phys. 1989, 91, 5011-5020. (b) Mieher, W.D.; Ho, W. J. Chem. Phys. 1990, 92, 5162-5164. (c) Hellsing, B.; Zhdanov, V.P. J. Electron Spectrosc. Relat. Phenom. 1993, 64, 563-569. (d) Zacharias, H.; Eichhorn, G.; Schliensing, R.; Al-Shamery, K. Appl. Phys. B Lasers Optics 1999, 68, 605-609.

(22) (a) Vondrak, T.; Zhu, X.Y. Phys. Rev. Lett. 1999, 82, 1967-1970. (b) Vondrak, T.; Zhu, X.Y. J. Phys. Chem. B 1999, 103, 4892-4899.

(23) (a) Hirata, S.; Head-Gordon, M.; Bartlett, R.J. J. Chem. Phys. 1999, 111, 10774-10786. (b) Hirata, S.; Grabowski, I.; Tobita, M.; Bartlett, R.J. Chem. Phys. Lett. 2001, 345, 475-480. (c) Hirata, S.; Podeszwa, R.; Tobita, M.; Bartlett, R.J. J. Chem. Phys. 2004, 120, 2581-2592.

(24) Minot, C.; Markovits, A. J. Mol. Struc. (Theochem.) 1998, 424, 119-134.

(25) Klüner, T.; Govind; N.; Yang, Y.A.; Carter, E.A. Phys. Rev. Lett. 2001, 86, 5954-5957.

(26) (a) Klüner, T.; Freund, H.J.; Freitag, J.; Staemmler, V. J. Mol. Catal. A-Chem. 1997, 119, 155-163. (b) Klüner, T.; Freund, H.J.; Staemmler, V.; Kosloff, R. Phys. Rev. Lett. 1998, 80, 5208-5211. (c) Klüner, T.; Thiel, S.; Freund, H.J.; Staemmler, V. Chem. Phys. Lett. 1998, 294, 413-418. (d) Thiel, S.; Pykavy, M.; Klüner, T.; Freund, H.J.; Kosloff, R.; Staemmler, V. J. Chem. Phys. 2002, 116, 762-773.

(27) Dovesi, R.; Saunderds, V.R.; Roetti, C.; Causá, M.; Harrison, N.M.; Orlando, R.; Aprá, E. CRYSTAL98 User's Manual, University of Torino, Torino, 1998.

(28) Muscat, J. Ph.D. Thesis, University of Manchester, Manchester, UK, 1999.

(29) Harrison, N.M.; Wang, X.G.; Muscat, J.; Scheffler, M. Faraday Discuss. 1999, 114, 305-312.

(30) Mackrodt, W.C.; Simson, E.A.; Harrison, N.M. Surf. Sci. 1997, 384, 192-200.

(31) (a) Bredow, T.; Aprà, E.; Catti, M.; Pacchioni, G. Surf. Sci. 1998, 418, 150-165. (b) Sanz, J.F.; ZicovichWilson, C.M. Chem. Phys. Lett. 1999, 303, 111-116.

(32) Zhu, C.; Byrd, R.H.; Lu, P.; Nocedal, J. Fortran Subroutines for Large Scale Bound Constrained Optimiza- tion; Dept. of Elec. Eng. and Comp. Sci, Northwestern University, Evanston, IL, 1994.

(33) Bartheland, P.K.; Durand, J.C.; Serafini, A. Mol. Phys. 1977, 33, 159-180.

(34) Hay, P.J.; Wadt, W.R. J. Chem. Phys. 1985, 82, 270283.

(35) (a) Muscat, J.; Harrison, N.M.; Thornton, G. Phys. Rev. B 1999, 59, 2320-2326. (b) Muscat, J.; Harrison, N.M.; Thornton, G. Phys. Rev. B 1999, 59, 15457-15463.

(36) Monkhorst, H.J.; Pack, J.D. Phys. Rev. B 1976, 13, 5188-5192. (b) Monkhorst, H.J.; Pack, J.D. Phys. Rev. $B$ 1976, 16, 1748-1749.

(37) Burdett, J.K.; Hughbanks, T.; Miller, G.J.; Richarson, J.W.; Smith, J.V. J. Am. Chem. Soc. 1987, 109, 36393646.

(38) Lindan, P.J.D.; Harrison, N.M.; Gillan, M.J.; White, J.A. Phys. Rev. B 1997, 55, 15919-15927.

(39) Wu, X.; Selloni, A.; Lazzeri, M.; Kayak, S.K. Phys. Rev. B 2003, 64, 241402-241405.

(40) Rasmussen, M.D.; Molina, L.M.; Hammer, B. J. Chem. Phys. 2004, 120, 988-997.

(41) Katz, G.; Kosloff, R.; Zeiri, Y. J. Chem. Phys. 2004, 3931-3948.

(42) de Lara-Castells, M.P.; Krause, J.L. J. Chem. Phys. 2003, 118-120, 5098-5105.

(43) (a) Bredow, T.; Pacchioni, G. Chem. Phys. Lett. 2002, 355, 417-423. (b) Bredow, T. Int. J. Quantum Chem. 1999, 75, 127-132.

(44) (a) Rittner, F.; Fink, R.; Boddenberg, B.; Staemmler, V. Phys. Rev. B 1998, 57, 4160-4171. (b) Casarin, M.; Maccato, M.; Vittadini, A. J. Phys. Chem. B 1998, 102, 10745-10752. (a) Casarin, M.; Maccato, M.; Vittadini, A. A. Surf. Sci. 1999, 142, 196-199.

(45) (a) Werner, H.J.; Knowles, P.J. J. Chem. Phys. 1985 , 82, 5053-5063. (b) Knowles, P.J.; Werner, H.J. Chem. Phys. Lett. 1985, 145, 259-267.

(46) Langhoff, S.R.; Davidson, E.R. Int. J. Quantum Chem. 1974, 8, 61-72.

(47) de Lara-Castells, M.P.; Roncero, O.; Krause, J.L.; Mitrushenkov, A.O. Unpublished.

(48) Malmqvist, P.A. Int. J. Quantum Chem. 1986, 30, 479494.

(49) see http://www.teokem.lu.se/molcas

(50) (a) Park, T.J.; Light, J.C. J. Chem. Phys. 1986, 85, 5870-5876. (b) Feagin, J.M. Quantum Methods with Mathematica; TELOS: Santa Clara, CA, 1994. (c) Gray, S.K. J. Chem. Phys. 1992, 96, 6543-6554.

(51) Fox, L. Numerical Solution of Two-Boundary Value Problems in Ordinary Differential Equations; Oxford University Press: London, 1957.

(52) Gadéa, F.X.; Berriche, H.; Roncero, O.; Villarreal, P.; Delgado-Barrio, G. J. Chem. Phys. 1997, 107, 1051510522.

(53) (a) Fano, U. Phys. Rev. 1961, 124, 1866-1878. (b) Beswick, J.A. In Dynamical processes in Molecular Physics; Delgado-Barrio, G., Ed.; IOP Pub. Ltd: New York, 1993.

(54) (a) Rose, T.S.; Rosker, M.J.; Zewail, A.H. J. Chem. 
Phys. 1989, 91, 7415-7436. (b) Engel, V.; Metiu, H. J. Chem. Phys. 1989, 91, 1596-1602.

(55) (a) de Lara-Castells, M.P.; Mitrushenkov, A.; Palmieri, P.; Quére, F.L.; Leonard, C.; Rosmus, P. Mol. Phys.
2000, 98, 1713-1727. (b) Mitrushenkov, A.O.; Palmieri, P.; Tarroni, R. In Science and Supercomputing at CINECA; Garofano, F.; Moretti, M.; Voli, M., Eds.; Cineca: Casalecchio di Reno, Italy, 2001, p 189. 